\title{
ON THE KRONECKER PRODUCTS OF IRREDUCIBLE REPRESENTATIONS OF THE $2 \times 2$ REAL UNIMODULAR GROUP. I
}

\author{
BY \\ LAJOS PUKÁNSZKY
}

Introduction. The purpose of the present paper is to determine the decomposition of the Kronecker product of two irreducible representations of the real $2 \times 2$ unimodular group into a continuous direct sum of irreducible representations.

The irreducible unitary representations of this group have been determined first by V. A. Bargmann [1] $\left.{ }^{1}\right)$, and those of the $2 \times 2$ complex unimodular group by I. M. Gel'fand and M. A. Naĭmark [3]. In both cases the list of these representations contains two continuous series; first, the principal continuous series, the members of which can be described by a pair $(m, \rho)$ of two variables, $m$ with a discrete, $\rho$ with a continuous range; and secondly, the representations of the exceptional interval, characterized by a single parameter, varying over a finite interval. In the real case in addition to these there exists a discrete series of representations characterized by integers. Concerning the representations of the exceptional interval it has been proved that they do not occur in the decomposition of the left regular representations of these groups into a continuous direct sum of irreducible representations.

The problem of finding the irreducible parts for the Kronecker product of two of these representations by the Reduction Theory of von Neumann [9] was taken up first by G. W. Mackey, in the complex case, for two factors taken from the principal series $[4 ; 5]$. W. F. Stinespring applied the same method to the discussion of the analogous case for the real group $\left({ }^{2}\right)$. Recently, M. A. Naimark attacked the same problem in the complex case, and gives a complete discussion of all possibilities $[10]\left({ }^{3}\right)$.

In Parts I, II, and III of the present work we give the decomposition of the product of any two irreducible unitary representations of the real $2 \times 2$ unimodular group. To sketch our method, we restrict ourselves, for the sake

Received by the editors September 6, 1960.

(1) When speaking of unitary representations in this paper, we mean always representations, which are strongly continuous. Bargmann's classification was performed under certain restrictive conditions (cf. $[1,(5.6)$, p. 601$])$. It has been proved, however, that these conditions are automatically fulfilled, cf. for instance, [6, p. 545].

(2) Oral communication.

( $\left.{ }^{3}\right)$ At the time being only the first part, dealing with the product of two representations of the principal series [10] is accessible to us. 
of simplicity, to the representations of the three-dimensional Lorentz group $L_{3}$; it is known that any representation of the $2 \times 2$ group is a single- or doublevalued representation of $L_{3}$. All notions used in the following will be described in appropriate detail in later sections. We describe $L_{3}$ as the connected component of unity in the group of all linear transformations of three-dimensional Euclidean space, which leave the form $x_{0}^{2}-x_{1}^{2}-x_{2}^{2}$ invariant. Furthermore, we denote the one-parameter subgroups of $L_{3}$ leaving $x_{0}, x_{1}$ and $x_{2}$ invariant by $G_{0}, G_{1}$ and $G_{2}$ resp. Given any continuous unitary representation $T_{a}$ of $L_{3}$ on a Hilbert space $H$, we denote the infinitesimal generators of the one-parameter groups of unitary transformations corresponding to $G_{i}$ by $H_{i}(i=0,1,2)$ resp. Forming the operator $Q^{\prime}=H_{1}^{2}+H_{2}^{2}-H_{0}^{2}$, one can show that its closure $Q$ is self-adjoint, and commutes with the operators $T_{a}\left(a \in L_{3}\right)$. In other words, $Q$ is affiliated with the center of the weakly closed operator ring generated by this representation. Hence in particular, in the case of an irreducible representation, it is of the form $q I$, where $q$ is a real number and $I$ is the unit operator. As Bargmann's list shows, the number $q$ determines the corresponding irreducible representation uniquely; its range is the interval $0<q<+\infty$ for the two continuous series, and $q=0,-1,-2, \cdots$ for the discrete series. Conversely, one can show that a representation, for which $Q$ has the form $q I$, is a multiple of the corresponding irreducible representation. Therefore, if we decompose the Hilbert space of the representation $T_{a}$ into a continuous direct sum of Hilbert spaces diagonalizing $Q$, then at the same time we get a decomposition of $T_{a}$ into a continuous direct sum of unitary representations, each of which is a multiple of some irreducible representation. From this, one can see that the problem of finding the irreducible components of a unitary representation essentially amounts to that of determining the spectrum of the corresponding operator $Q$. To decide which members of the discrete series occur in the decomposition, is made easy by the fact that they appear always as discrete summands. Therefore in the following we deal with the problem of finding the irreducible components belonging to one of the continuous series. Let $T_{a}$ be an irreducible representation of this kind. Since $G_{0} \subset L_{3}$ is the group of rotations of the unit circle, $T_{a}$, when restricted to $G_{0}$, breaks up into a discrete direct sum of one-dimensional representations, and one can show that the trivial representation occurs exactly once. For an irreducible representation of the discrete series the trivial representation does not occur at all. From what has just been said, it is clear that if for a representation $T_{a}$ we have $Q=q I(q>0)$, then, denoting by $\mathfrak{M}_{0}$ the subspace of all vectors which are left invariant by the restriction of $T_{a}$ to $G_{0}, T_{a}$ is a $\operatorname{dim} \mathfrak{M}_{0}$ times multiple of the irreducible representation belonging to $q$. From this we can deduce the following general rule. Let $T_{a}$ be an arbitrary unitary representation of $L_{3}$ on the Hilbert space $H$; we form the corresponding operator $Q$ and define the subspace $\mathfrak{M}_{0}$ as above. $\mathfrak{M}_{0}$ obviously reduces $Q$; we denote its part in it by $Q_{0}$. One knows that one can represent $\mathfrak{M}_{0}$ in a unique 
manner as a sum $\sum_{\nu=1}^{\infty} \oplus \mathfrak{M}_{\nu}$ such that each $\mathfrak{M}_{\nu}$ reduces $Q_{0}$, and the part $Q_{0}^{(\nu)}$ of $Q_{0}$ in $\mathfrak{M}_{\nu}$ is a $\nu$-fold copy of an operator with a simple spectrum; finally, the spectra of $Q_{0}^{(\nu)}$ and $Q_{0}^{(\mu)}$ for $\nu \neq \mu$ are pairwise disjoint $\left(^{4}\right)$. In this case $T_{a}$ is a direct sum of two representations $T_{a}^{d}$ and $T_{a}^{c}$. $T_{a}^{d}$ is a discrete direct sum of irreducible representations of the discrete series and of the trivial representation; $T_{a}^{c}$ is a direct sum of a sequence $T_{a}^{(\nu)}(\nu=1,2, \cdots)$ of unitary representations, such that $T_{a}^{(\nu)}$ is a continuous direct sum, with respect to a weight function equivalent to the spectrum of $Q_{0}^{(\nu)}$, of $\nu$-fold copies of irreducible representations from the continuous series.

Up to now we have been discussing the case of an arbitrary unitary representation of $L_{3}$, and have arrived at the conclusion that the main difficulty in determining its irreducible components is that of finding the spectral properties of $Q_{0}$ (with the notations used above). Turning, for example, to the particular case of the Kronecker product of two irreducible representations of the continuous series of $L_{3}$, and making use of Bargmann's description of these representations in terms of function spaces, we have to deal, among other things, with problems of the following type. Consider an integrable positive-definite function $K(\phi)$ on the circumference of the unit circle; suppose that $\int_{0}^{2 \pi} \int_{0}^{2 \pi} K(\phi-\psi) f(\phi) \overline{f(\psi)} d \phi d \psi$ is positive for every continuous function $f(\phi)$. Then define an inner product for any pair $f(\phi)$ and $g(\psi)$ of continuous functions by $(f, g)=\int_{0}^{2 \pi} \int_{0}^{2 \pi} K(\phi-\psi) f(\phi) \overline{g(\psi)} d \phi d \psi$. In this fashion we get a preHilbert space; denote its completion by $H$. Then we are to investigate the spectrum (including multiplicity) of a self-adjoint operator, obtained as the closure of a certain linear differential operator of second order defined on the sufficiently smooth elements of $H$ (cf. (B) in Chapter II of the present Part I). In a similar fashion, when discussing the decomposition of a product, with one factor of type $D_{l}^{+}$, the other of $D_{k}^{-},\left(^{5}\right)$ we shall have to consider differential operators of second order on certain Hilbert spaces, formed from analytic functions on the unit disk.

As a feature common to all cases of Kronecker products we wish to note the following two facts: (a) either no representation from the principal continuous series occurs, or every representation from the principal continuous series occurs, with a weight function equivalent to the Lebesgue measure, (b) representations of the exceptional interval appear as discrete direct summands only.

The purpose of the present Part I is the discussion of products with factors from the two continuous series of $L_{3}$. Its plan is as follows. In Chapter I we give a detailed discussion of the method, outlined above, to obtain the irreducible components of any unitary representation of the $2 \times 2$ real uni-

(4) We say that the spectra of two self-adjoint operators $A_{1}$ and $A_{2}$ on the Hilbert space $H$, with the spectral resolutions $E_{\lambda}^{(1)}$ and $E_{\lambda}^{(2)}$ are disjoint, if the measures corresponding to the functions $\left\|E_{\lambda}^{(1)} f\right\|^{2}$ and $\left\|E_{\lambda}^{(2)} g\right\|^{2}$ of bounded variation are disjoint for any choice of $f, g \in H$.

(b) For these cf. the end of I.A. 
modular group. The fact that we have been anxious to give, as far as this is possible, a self-contained treatment, explains its length. In §A of Chapter II we determine the irreducible components belonging to the discrete series; in $\S \mathrm{B}$ those of the continuous series. This is achieved through discussion of the operator $Q_{0}$, as indicated above.

Part II will treat those products in which at least one factor belongs to the discrete series. Finally, Part III will discuss the product of two representations belonging to the continuous series, one or both of the factors being double-valued representations of $L_{3}$. These will be published later.

Most of the results of the present paper were obtained during the author's stay at the University of Chicago in January-March, 1958. The author is indebted to Professor I. E. Segal for proposing the subject of the present research.

\section{Chapter I. Preliminaries}

A. Irreducible representations of $\mathfrak{S}$. In this section we summarize certain results concerning general representation theory, and in particular, concerning irreducible representations of the $2 \times 2$ real unimodular group.

Following Bargmann, we consider, instead of the group of all $2 \times 2$ real unimodular matrices, the isomorphic group of all $2 \times 2$ complex matrices of the form

$$
\left(\begin{array}{ll}
\alpha & \beta \\
\bar{\beta} & \bar{\alpha}
\end{array}\right), \quad|\alpha|^{2}-|\beta|^{2}=1 .
$$

We denote this group by $\mathfrak{\subseteq}$. We observe that $\subseteq$ is locally isomorphic with the three-dimensional Lorentz group $L_{3}$, by which we mean the connected component of unity in the group of all nonsingular linear transformations of $R^{3}$, leaving the form $x_{0}^{2}-x_{1}^{2}-x_{2}^{2}$ invariant. As a matter of fact, $L_{3}$ is isomorphic to the factor group of $\subseteq$ according to the subgroup of order 2 consisting of $\pm e$, where $e$ is the unit matrix.

Next we consider the following one-parameter subgroups of $\mathfrak{S}$ :

$$
\begin{aligned}
g_{0}(t)=\left(\begin{array}{ll}
e^{-i t / 2} & 0 \\
0 & e^{i t / 2}
\end{array}\right), \quad g_{1}(t)=\left(\begin{array}{cc}
\operatorname{ch} t / 2 & i \operatorname{sh} t / 2 \\
-i \operatorname{sh} t / 2 & \operatorname{ch} t / 2
\end{array}\right), \\
g_{2}(t)=\left(\begin{array}{cc}
\operatorname{ch} t / 2 & \operatorname{sh} t / 2 \\
\operatorname{sh} t / 2 & \operatorname{ch} t / 2
\end{array}\right) \quad(-\infty<t<+\infty) .
\end{aligned}
$$

Let us suppose that $T_{a}$ is a continuous unitary representation of the group $\subseteq$ on the Hilbert space $H$. Defining the self-adjoint operators $H_{j}(j=0,1,2)$ on $H$ by

$$
T_{g_{j}(t)}=\exp \left(-i H_{j} t\right) \quad(j=0,1,2)
$$

one easily verifies, by aid of the reasonings [2, pp. 601-602], that the operator 
$Q^{\prime}=H_{1}^{2}+H_{2}^{2}-H_{0}^{2}$ formally commutes with all the operators of the representation $T_{\alpha}$, hence by the theorem in [12] it is essentially self-adjoint, that is, it is densely defined and its closure $Q$ is self-adjoint on $H$. We call $Q$ the Casimir operator belonging to our representation. In particular, if $T_{a}$ is irreducible, then $Q$ is of the form $q I$, where $q$ is real, and $I$ denotes the unit operator on $H$. In the following we reproduce Bargmann's classification of all nontrivial irreducible unitary representations of $\subseteq[1$, p. 609], with minor modifications in the notation $\left(^{6}\right)$. Since the one-parameter subgroup $g_{0}$ is compact, the operator $H_{0}$ has a pure point spectrum consisting of integers or half-integers (we parametrize $g_{0}$ in such a way, that $g_{0}(2 \pi)=-e, g_{0}(4 \pi)=e$, where $e$ is the unit in (5). It turns out that each eigenvalue occurs with the multiplicity one. Then the set of all nontrivial irreducible representations of $\subseteq$ can be listed as follows:

(1) Principal continuous series

(a) integral case $C_{q}^{0}(q \geqq 1 / 4 ; m=0, \pm 1, \pm 2, \cdots)$,

(b) half integral case $C_{q}^{1 / 2}(q>1 / 4 ; m=0, \pm 1 / 2, \pm 3 / 2, \cdots)$.

(2) Continuous series; exceptional interval

$$
E_{q}(0<q<1 / 4 ; m=0, \pm 1, \pm 2, \cdots) .
$$

(3) Discrete series

(a) minimal $m: D_{\boldsymbol{k}}^{+}(q=k(1-k) ; m=k, k+1, \cdots ; k=1 / 2,1,3 / 2, \cdots)$,

(b) maximal $m: D_{\boldsymbol{k}}^{-}(q=k(1-k) ; m=-k,-k-1, \cdots ; k=1 / 2,1$, $3 / 2, \cdots)$.

An irreducible representation $T_{a}$ with $T_{-e}=I$, is a single-valued representation of $L_{3}$. From among the representations listed above $C_{q}^{0}(1 / 4 \leqq q<+\infty)$, $E_{q}(0<q<1 / 4)$ and $D_{k}^{+}, D_{k}^{-}$, where $k$ is an integer, possess this property. We see that the conditions $T_{-e}=I$ and $0<q<+\infty$ determine an irreducible representation of $L_{3}$ uniquely.

B. Decomposition into irreducible representations. The purpose of the subsequent considerations is to show that the problem of finding the decomposition of a continuous unitary representation of $\subseteq$ into irreducible parts can to a large extent be reduced to the investigation of the spectral properties of the Casimir operator associated with this representation (cf. $\S \mathrm{A}$ ). Though the following statements could be directly obtained from known results (cf. beside what is going to be quoted in the course of the proof $[6, \S \S 4-6$; 7 , Theorem ]), for the convenience of the reader we prefer to give a complete discussion, making use of the present special situation. Here we shall need the more elementary part of the Reduction Theory only, for which we refer the reader to $[9, \S \$ 1-14]$.

(6) In view of the particular role played by the representations of the exceptional interval (cf. $[1, \S 8]$ ) in the decomposition of the Kronecker products (cf. Introduction) we denote them by $E_{q}$ instead of $C_{q}^{0}(0<q<1 / 4)$ as used by Bargmann. 
B1. Let $T_{a}$ be a continuous unitary representation of $\subseteq$ given on the separable Hilbert space $H$. Then there exists a decomposition, with respect to a weight function $\sigma(\lambda)$ over the real line, of this Hilbert space into a continuous direct sum of Hilbert spaces $H_{\lambda}$, a continuous unitary representation $T_{a}(\lambda)$ of $\subseteq$ on $H_{\lambda}$ such that (a) for each $a \in \mathbb{S}$ we have $T_{a}=\sum T_{a}(\lambda)$, (b) the Casimir operator $Q(\lambda)$ of $T_{a}(\lambda)$ equals $\lambda I$.

First we suppose that for our representation $T_{-e}=I$.

We denote the Lie algebra of $\subseteq$ by $\mathfrak{A}$ (cf. [1, 4.b, p. 595]). For $\chi \in \mathfrak{A}$ we put $g_{\chi}(t)=\exp \chi t$, and we define $H_{\chi}$ through the condition

$$
T_{g_{\chi}(t)}=\exp \left(-i H_{\chi} t\right) \text {. }
$$

We denote the element of $\mathfrak{A}$ corresponding to the subgroups $g_{i}(t)$ (cf. $\S \mathrm{A}$ ) by $\chi_{i}$ resp., and as before, we put $H_{i}=H_{\chi_{i}}(i=0,1,2)$. By Theorem 3.1 in [11] there exists a dense linear manifold $\mathfrak{B} \subset H$, such that (a) $\mathfrak{B}$ is in the intersection of the domains of definition of all $H_{\chi}(\chi \in \mathfrak{A})$, and the restriction $H_{\chi}^{\prime}$ of $H_{\chi}$ to $\mathfrak{B}$ is essentially self-adjoint, that is $H_{\chi}^{\prime * *}=H_{\chi}$, (b) we have $H_{\chi} \mathfrak{B}$ $\subset \mathfrak{B}(\chi \in \mathfrak{A})$ and $T_{a} \mathfrak{B} \subseteq \mathfrak{B}(a \in \mathfrak{S})$, (c) the mapping $\chi \rightarrow i H_{\chi}^{\prime}$ gives a representation of $\mathfrak{A}$ by the essentially skew-adjoint operators $i H_{\chi}^{\prime}$, finally (d) the operator $H_{1}^{2}+H_{2}^{2}-H_{0}^{2}$ is essentially self-adjoint on $\mathfrak{B}$ and commutes with $T_{a}(a \in \mathfrak{S})$; its closure, commuting again with $T_{a}$, is the Casimir operator of our representation; and will be denoted again by $Q$ (cf. [1, 2.c., p. 587, 5.e., p. 601], and [12, Theorem]). If $P$ is any projection commuting with $T_{a}(a \in \mathfrak{S})$, and $\mathfrak{M}$ the corresponding subspace, then it is clear that the Casimir operator of the restriction of $T_{a}$ to $\mathfrak{M}$ is the restriction of $Q$ to $\mathfrak{M}$. Hence without loss of generality we may assume in the following that $(\alpha) Q$ is bounded, $(\beta) Q$ does not possess nontrivial eigenvectors. For if $\mathfrak{M}_{\lambda}$ is the eigenspace belonging to the eigenvalue $\lambda$ of $Q$, then it is invariant under $T_{a}$, and the Casimir operator of the part of $T_{a}$ in $\mathfrak{M}_{\lambda}$ is $\lambda I$.

Let $D_{i}$ be the domain of the operators $H_{i}(i=0,1,2)$ resp. We show that $D_{0} \subset D_{1} \cap D_{2}$. Indeed, if $f \in D_{0}$, by (a) above we can find a sequence $f_{n}$ of elements in $\mathfrak{B}$, such that $\lim _{n \rightarrow \infty} f_{n}=f$ and $\lim _{n \rightarrow \infty} H_{0} f_{n}=H_{0} f$. But we have

$$
\left\|H_{0}\left(f_{n}-f_{m}\right)\right\|^{2}+\left(Q\left(f_{n}-f_{m}\right), f_{n}-f_{m}\right)=\left\|H_{1}\left(f_{n}-f_{m}\right)\right\|^{2}+\left\|H_{2}\left(f_{n}-f_{m}\right)\right\|^{2}
$$

and hence, by virtue of our assumption concerning the continuity of $Q$, we see that the sequences $\left\{H_{i} f_{n}\right\}(i=1,2)$ converge too, along with $\left\{f_{n}\right\}$, which proves that $f \in D_{1} \cap D_{2}$.

Since the subgroup $g_{0}$ is compact, and since $T_{-e}=I$, we have

$$
T_{g_{0}(t)}=\sum_{(n)} e^{-i n t} P_{n}
$$

where the $P_{n}(n=0, \pm 1, \pm 2, \cdots)$ are pairwise orthogonal projections, with a sum equal to $I$; we denote the subspace corresponding to $P_{n}$ by $\mathfrak{M}_{n}$. Since 
$\mathfrak{M}_{n} \subset D_{0}$, we have $\mathfrak{M}_{n} \subset D_{1} \cap D_{2}$; in what follows we show that putting $H_{+}$, $H_{-}$for the closures of the operators $H_{1}+i H_{2}, H_{1}-i H_{2}$ resp., we have $H_{-} \mathfrak{M}_{n}$ $\subset \mathfrak{M}_{n-1}, H_{+} \mathfrak{M}_{n} \subseteq \mathfrak{M}_{n+1}(n=0,1,2, \cdots)$. What follows is essentially an adaptation of the reasoning of $5 f$ on $\left[1\right.$, p. 603]. If $\chi=\alpha^{\prime} \chi^{\prime}+\alpha^{\prime \prime} \chi^{\prime \prime}\left(\alpha^{\prime}, \alpha^{\prime \prime}\right.$ real, and $\left.\chi^{\prime}, \chi^{\prime \prime} \in \mathfrak{A}\right)$, then $H_{\chi}=\alpha^{\prime} H_{\chi^{\prime}}+\alpha^{\prime \prime} H_{\chi^{\prime \prime}}$ on $\mathfrak{B}$, and since the restriction of any $H_{\chi}$ to $\mathfrak{B}$ is essentially self-adjoint, the same relation holds for any $f \in D_{\boldsymbol{x}} \cap D_{\chi^{\prime \prime}}$. We have

$$
\begin{aligned}
& \bar{\chi}=g_{0}(t) \chi_{1}\left[g_{0}(t)\right]^{-1}=\cos t_{x_{1}}+\sin t_{\chi_{2}}, \\
& \overline{\bar{\chi}}=g_{0}(t) \chi_{2}\left[g_{0}(t)\right]^{-1}=-\sin t_{x_{1}}+\cos t_{\chi}
\end{aligned}
$$

and putting $U_{t}=\exp \left(-i t H_{0}\right)$

$$
H_{\bar{x}}=U_{t} H_{1} U_{-t}, \quad H_{\overline{\bar{x}}}=U_{t} H_{2} U_{-t} .
$$

Therefore, if $g \in \mathfrak{M}_{n}$, then

$$
\begin{aligned}
& e^{i n t} U_{t} H_{1} g=\cos t H_{1} g+\sin t H_{2} g, \\
& e^{i n t} U_{t} H_{2} g=-\sin t H_{1} g+\cos t H_{2} g
\end{aligned}
$$

and therefore

$$
\begin{aligned}
& U_{t} H_{+} g=e^{-i(n+1) t} H_{+} g \\
& U_{t} H_{-} g=e^{-i(n-1) t} H_{-} g .
\end{aligned}
$$

Hence

$$
\begin{aligned}
& \frac{1}{t}\left(U_{t}-I\right) H_{+} g=\frac{1}{t}\left(e^{-i(n+1) t}-1\right) H_{+} g, \\
& \frac{1}{t}\left(U_{t}-I\right) H_{-} g=\frac{1}{t}\left(e^{-i(n-1) t}-1\right) H_{-} g .
\end{aligned}
$$

Since in each case the right-hand side tends to a limit, if $t \rightarrow 0$, we have $H_{+} g$, $H_{-} g \in D_{0}$, and

$$
\begin{aligned}
& H_{0} H_{+} g=(n+1) H_{+} g, \\
& H_{0} H_{-} g=(n-1) H_{-} g
\end{aligned}
$$

which proves our assertion.

Furthermore, we have

$$
g_{2}(t) \chi_{1}\left[g_{2}(t)\right]^{-1}=\operatorname{sh} t \chi_{0}+\operatorname{ch} t \chi_{1}
$$

We put $U_{l}=\exp i H_{2} t$. If $g$ is in the domain of the operators $H_{i} H_{j}(i, j=0,1,2)$, then

$$
H_{1} U_{t} g=U_{t}\left(\operatorname{sh} t H_{0}+\operatorname{ch} t H_{1}\right) g ;
$$

hence 


$$
H_{1}\left\{\frac{1}{t}\left(U_{t}-I\right) g\right\}=t^{-1} \operatorname{sh} t U_{t} H_{0} g+t^{-1}\left(\operatorname{ch} t U_{t}-I\right) H_{1} g .
$$

If $t \rightarrow 0$, then the right-hand side tends to $H_{0} g+i H_{2} H_{1} g$. Therefore, in particular, whenever $g \in \mathfrak{M}_{n}$, we have

$$
\left(H_{1} H_{2}-H_{2} H_{1}\right) g=-i H_{0} g
$$

and

$$
\left(H_{-} H_{+}-H_{+} H_{-}\right) g=2 H_{0} g
$$

and finally, by definition

$$
\left(H_{-} H_{+}+H_{+} H_{-}\right) g=2\left(Q+H_{0}^{2}\right) g \text {. }
$$

In what follows, we denote the operator ring consisting of all bounded functions of $Q$ by $R$. Let us consider the decomposition of $H$, with respect to the weight function $\sigma(\lambda)$, into a continuous direct sum of Hilbert spaces $H_{\lambda}$, to which the ring $R$ belongs. For this we write

$$
H=\int_{R^{1}} \oplus H_{\lambda} d \sigma
$$

We may obviously assume that it is the multiplication by $\lambda$ which corresponds to $Q$. We put $P_{0}=\sum P_{0}(\lambda)$; then $\operatorname{dim} P_{0}(\lambda)$ is a measurable function of $\lambda$, and it is positive almost everywhere. For otherwise we would have a projection $P \in R$ such that $P_{0} P=0$. Let $n$ be the integer with smallest absolute value, for which $P_{n} P \neq 0$. We suppose that $n>0$; the opposite sign can be treated in an analogous way. If $f \neq 0, P_{n} P f=f$, then $H_{-} f=0$, since $H_{-} f \in \mathfrak{M}_{n-1}$ and $P$ commutes with $H_{-}$and $P_{n-1}$. But using relations (*) we get

$$
0=H_{+} H_{-} f=(Q+n(n-1) I) f=0 .
$$

In other words, $f$ is an eigenvector of $Q$, hence $f=0$, since we assumed that $Q$ does not have nontrivial eigenvectors, which gives a contradiction.

In the following we suppose, by possibly restricting our representation $T_{a}$ to a subspace, the projection of which belongs to $R$, that $\operatorname{dim} P_{0}(\lambda)$ $\equiv m>0(m=1,2, \cdots,+\infty)$. Then we can find $m$ vectors $e^{(i)}(i=1,2, \cdots, m)$ in $\mathfrak{M}_{0}$, such that if

$$
e^{(i)}=\int_{R^{1}} e^{(i)}(\lambda)(d \sigma(\lambda))^{1 / 2}
$$

then $e^{(i)}(\lambda)$ form a complete orthonormal system in the subspace $\mathfrak{M}_{0}(\lambda)$ of $H_{\lambda}$ belonging to the projection $P_{0}(\lambda)$. We put

$$
f_{0}^{(i)}=e^{(i)}, \quad f_{8}^{(i)}=H_{+}^{8} e^{(i)}, \text { and } f_{-8}^{(i)}=H_{-}^{8} e^{(i)}
$$


and

$$
f_{s}^{(i)}=\int f_{s}^{(i)}(\lambda)(d \sigma(\lambda))^{1 / 2} \quad(s=1,2, \cdots ; i=1,2, \cdots, m) .
$$

We are going to prove that $\left(f_{s}^{(i)}(\lambda), f_{t}^{(j)}(\lambda)\right) \equiv 0$, a.e. with respect to $\sigma$, if either $i \neq j$ or $s \neq t$. For this it is enough to show that under this assumption $\left(A f_{s}^{(i)}, f_{t}^{(j)}\right)=0$ for any $A \in R$. If $s \neq t$ this is evident, because $\mathfrak{M}_{s}$ and $\mathfrak{M}_{t}$ are orthogonal and each of them reduces every operator of $R$. To prove that $\left(A f_{k}^{(i)}, f_{k}^{(j)}\right)=0$ observe that this is true for $k=0$ since $f_{0}^{(i)}=e^{(i)}$ and by virtue of our choice of $e^{(i)}(i=1,2, \cdots, m)$. Suppose now that our statement has already been proved for $k=1,2, \cdots, s$. Then using relations $\left({ }^{*}\right)$ we get

$$
\begin{aligned}
\left(A f_{s+1}^{(i)}, f_{s+1}^{(j)}\right) & =\left(A H_{+} f_{s}^{(i)}, H_{+} f_{s}^{(j)}\right)=\left(A H_{-} H_{+} f_{s}^{(i)}, f_{s}^{(j)}\right) \\
& =\left(A Q f_{s}^{(i)}, f_{s}^{(j)}\right)+s(s+1)\left(f_{s}^{(i)} f_{s}^{(j)}\right)=0 .
\end{aligned}
$$

Similar reasoning applies if $s<0$. Moreover we have for any $A \in R$

$$
\left(A f_{s+1}^{(i)}, f_{s+1}^{(i)}\right)=\left(A(Q+s(s+1) I) f_{s}^{(i)}, f_{8}^{(i)}\right) .
$$

Incidentally, this gives that $Q \geqq 0$, because otherwise we would have for an appropriate $A>0, A \in R$ and $s=0,\left\|A^{1 / 2} f_{1}^{(i)}\right\|^{2}=\left(Q A f_{0}^{(i)}, f_{0}^{(i)}\right)<0$. Therefore in the following we may assume that the $\sigma$ measure of the half-line $(-\infty, 0)$ is 0 . Putting $\rho_{j}(\lambda) \equiv(\lambda+j(j-1))(j=1,2, \cdots ; \lambda \geqq 0)$, and $r_{s}(\lambda) \equiv \prod_{j=1}^{s} \rho_{j}^{1 / 2}(\lambda)$ $(s=1,2, \cdots)$ we have

$$
\left\|f_{s}^{(i)}(\lambda)\right\| \equiv r_{s}(\lambda)
$$

almost everywhere with respect to $\sigma$. Similarly

$$
\left(A f_{-s-1}^{(i)}, f_{-s-1}^{(i)}\right)=\left(A(Q+s(s+1) I) f_{-s}^{(i)}, f_{-s}^{(i)}\right) .
$$

Hence we get

$$
\left\|f_{-s}^{(i)}(\lambda)\right\| \equiv r_{s}(\lambda) \quad \text { almost everywhere. }
$$

Summing up, after omitting a set of $\sigma$-measure 0 , and putting

$$
\begin{aligned}
e_{0}^{(i)}(\lambda) & =e^{(i)}(\lambda), \\
e_{s}^{(i)}(\lambda) & =\frac{1}{r_{s}(\lambda)} f_{s}^{(i)}(\lambda), \\
e_{-s}^{(i)}(\lambda) & =\frac{1}{r_{8}(\lambda)} f_{-s}^{(i)}(\lambda) \quad(s=1,2, \cdots ; i=1,2, \cdots, m),
\end{aligned}
$$


we get that for each $\lambda$ the sequence $\left\{e_{s}^{(i)}(\lambda)\right\}$ forms an orthonormal system in the space $H_{\lambda}$. We are going to prove now that for each $\lambda$ this system is complete. To see this, it is obviously enough to show that the system $\left\{A f_{s}^{(i)}\right\}(A \in R)$ spans the Hilbert space $H$. But this follows from the fact that for a fixed $s$ the set $\left\{A f_{s}^{(i)}\right\}(A \in R ; i=1,2, \cdots, m)$ spans the space $\mathfrak{M}_{s}$. To prove this, we proceed again by induction. For $s=0$ the statement is clearly correct. Let us suppose that it holds true for $0 \leqq k \leqq s$. If $g \neq 0$, $g \in \mathfrak{M}_{s+1}$ and $\left(A f_{s+1}^{(i)}, g\right)=0(A \in R, i=1,2, \cdots, m)$, then $\left(A f_{s}^{(i)}, H_{-} g\right)=0$, and hence, by virtue of our assumption, $H_{-} g=0$. But as we know, this implies that $g$ is a nontrivial eigenvector of $Q$, which we excluded. Similar argument applies to a negative $s$.

Now let us define the operators $H_{0}^{(j)}(\lambda), H_{+}^{(j)}(\lambda)$ and $H_{-}^{(j)}(\lambda)(j=1,2, \cdots, m)$ in the Hilbert space $H_{\lambda}(\lambda \geqq 0)$ by

$$
\begin{aligned}
& H_{0}^{(j)}(\lambda) e_{s}^{(j)}(\lambda)=s e_{s}^{(j)}(\lambda) \\
& H_{+}^{(j)}(\lambda) e_{s}^{(j)}(\lambda)=(\lambda+s(s+1))^{1 / 2} e_{s+1}^{(j)}(\lambda) \\
& H_{-}^{(j)}(\lambda) e_{s}^{(j)}(\lambda)=(\lambda+s(s-1))^{1 / 2} e_{s-1}^{(j)}(\lambda)
\end{aligned}
$$

and

$$
H_{-}^{(j)}(\lambda) e_{s}^{(i)}(\lambda)=H_{+}^{(j)}(\lambda) e_{s}^{(i)}(\lambda)=H_{-}^{(j)}(\lambda) e_{s}^{(i)}(\lambda)=0
$$

if $i \neq j(s=0, \pm 1, \pm 2, \cdots)$. We denote the closure of the operators $(1 / 2)\left(H_{+}^{(j)}(\lambda)+H_{-}^{(j)}(\lambda)\right)$ and $-(i / 2)\left(H_{+}^{(j)}(\lambda)-H_{-}^{(j)}(\lambda)\right)$ by $H_{1}^{(j)}(\lambda)$ and $H_{2}^{(j)}(\lambda)$ resp. Now we use the result of $[1, \S \S 6-8]$, according to which there exists a continuous irreducible unitary representation $T_{a}^{(j)}(\lambda)$ of $\subseteq$ in the subspace of $H_{\lambda}$ generated by the vectors $e_{s}^{(j)}(\lambda)$ for a fixed $j$, such that

$$
T_{g_{i}(t)}^{(j)}(\lambda)=\exp \left(-i t H_{i}^{(j)}(\lambda)\right)
$$$$
(i=0,1,2)
$$

and the corresponding Casimir operator equals $\lambda$ times unity. We put $H_{i}(\lambda)$ $=\sum_{j=1}^{m} H_{i}^{(j)}(\lambda)$; then $H_{i}(\lambda)$ is the infinitesimal generator for the oneparameter subgroup $T_{g_{i}(t)}(\lambda)(i=0,1,2)$ resp., where $T_{a}(\lambda)$ is the representation of $\mathfrak{S}$ on $H_{\lambda}$, which is a direct sum of the pairwise unitary-equivalent representations $T_{a}^{(j)}(\lambda)(j=1,2, \cdots, m)$. We are going to show that for each $a \in \mathbb{S}$ we have $T_{a}=\sum T_{a}(\lambda)$. Since every element $a \in \mathfrak{S}$ can be represented in the form $g_{0}\left(t_{1}\right) g_{2}\left(t_{2}\right) g_{0}\left(t_{3}\right)[1,4.12$, p. 595], it is enough to show that

$$
\exp \left(-i t H_{j}\right)=\sum \exp \left(-i t H_{j}(\lambda)\right)
$$

for $j=0,2$. We begin with $j=2$. Observe first that putting

$$
e_{s}^{(i)}=\int e_{s}^{(i)}(\lambda)(d \sigma(\lambda))^{1 / 2}
$$


we have

$$
H_{2}^{n} e_{s}^{(i)}=\int\left[H_{2}(\lambda)\right]^{n} e_{s}^{(i)}(\lambda)(d \sigma(\lambda))^{1 / 2} \quad(n=1,2, \cdots) .
$$

Now using the fact that the support of $\sigma$ is contained in a finite interval $[0, \Lambda]$ (since we assumed that $Q$ is bounded), and taking in view the expression for $\rho_{s}(\lambda)$, we can conclude that for $a>1$ there exists a $K>0$, not depending on $n$ and $\lambda$, such that

$$
\left\|\left[H_{2}(\lambda)\right]^{n} e_{s}^{(j)}(\lambda)\right\|<K a^{n} \quad(0 \leqq \lambda \leqq \Lambda),
$$

and

$$
\left\|\left[H_{2}\right]^{n} e_{s}^{(j)}\right\|<K a^{n} \quad(n=0,1,2, \cdots) .
$$

But this proves that the series

$$
\sum_{n=0}^{\infty} \frac{(-i t)^{n}\left[H_{2}(\lambda)\right]^{n} e_{s}^{(j)}}{n !}
$$

and

$$
\sum_{n=0}^{\infty} \frac{(-i t)^{n}\left[H_{2}\right]^{n} e_{s}^{(j)}}{n !}
$$

strongly converge to $\exp \left(-i t H_{2}(\lambda)\right) e_{s}^{(j)}(\lambda)$ and $\exp \left(-i t H_{2}\right) e_{s}^{(i)}$ resp., from which we conclude easily that

$$
\begin{aligned}
\exp \left(-i t H_{2}\right) e_{s}^{(j)}=\int & \exp \left(-i t H_{2}(\lambda)\right) e_{s}^{(j)}(\lambda)(d \sigma)^{1 / 2} \\
& (j=1,2, \cdots, m ; s=0, \pm 1, \pm 2, \cdots) .
\end{aligned}
$$

This implies the same relation for $A e_{s}^{(i)}(A \in R)$ instead of $e_{s}^{(i)}$; but since elements of this form span the space, this is true for any $f \in H$. The fairly simple proof for the case $j=0$ we leave to the reader.

Hence the statement, announced at the beginning of $\mathrm{B} 1$, has been proved for the case $T_{-e}=I$. If $T_{-e}=-I$, the proof is very much similar. Here we have

$$
T_{o_{0}(t)}=\sum_{(n)} e^{-i(n+1 / 2) t} P_{n+1 / 2}
$$

We denote the eigenspace of $H_{0}$ belonging to the eigenvalue

$$
n+1 / 2 \quad(n=0, \pm 1, \pm 2, \cdots)
$$

by $\mathfrak{M}_{n+1 / 2}$. After having performed the same reductions as before, we choose 
$m$ vectors $e^{(i)}(i=1,2, \cdots, m)$ in $\mathfrak{M}_{1 / 2}$ and define

$$
f_{1 / 2}^{(i)}=e^{(i)}, \quad f_{1 / 2+8}^{(i)}=\left(H_{+}\right)^{s} f_{1 / 2}^{(i)}, \quad f_{1 / 2-8}^{(i)}=\left(H_{-}\right)^{s} f_{1 / 2}^{(i)} \quad(s=1,2, \cdots) .
$$

Here we can show that not only $Q \geqq 0$, but even $Q \geqq I / 4$. The sequence $\left\{f_{1 / 2+8}\right\}$ has the same properties as the sequence $\left\{f_{s}\right\}$ had before, with the only exception that putting

$$
\rho_{s}(\lambda) \equiv\left(\lambda-1 / 4+s^{2}\right) \quad(s=1,2, \cdots ; \lambda>1 / 4)
$$

and

$$
r_{s}(\lambda) \equiv \prod_{j=1}^{s} \rho_{j}^{1 / 2}(\lambda), \quad r_{s}^{\prime}(\lambda) \equiv \prod_{j=0}^{8-1} \rho_{j}^{1 / 2}(\lambda)
$$

we have

$$
\left\|f_{1 / 2+s}(\lambda)\right\| \equiv r_{s}(\lambda), \quad\left\|f_{1 / 2-s}(\lambda)\right\| \equiv r_{s}^{\prime}(\lambda) .
$$

The definition of the operators $H_{+}^{(j)}(\lambda), H_{-}^{(j)}(\lambda)$ and $H_{0}^{(j)}(\lambda)$ is as before, only the range of $s$ must be replaced by the half-integers, in the corresponding formulas. Otherwise the same argument literally applies to the present case.

Finally, for any representation $T_{a}$, one can find a projection $P$ commuting with it, such that $P T_{-e}=P$ and $(I-P) T_{-e}=-(1-P)$ which settles the general case. We leave the proof of this statement to the reader. Since for the Kronecker product of two irreducible representations $T_{-e}= \pm I$, actually we shall make use of these cases only.

B2. Let $T_{a}$ be a unitary representation of $\mathfrak{S}$ on the Hilbert space $H$, such that its Casimir operator $Q$ is of the form $q I$, where $q$ is a real number. Then $T_{a}$ is a direct sum of a finite number of representations $T_{a}^{(i)}(i=1,2, \cdots, m)$, each of which is a multiple of some irreducible representation. The irreducible representations belonging to $T_{a}^{(i)}, T_{a}^{(j)}, i \neq j$, are inequivalent.

It will turn out that the maximum value of $m$ is 3 .

Since most of the reasonings needed in the following have already been used, we can be quite short. We use the same notations as before.

Suppose first that $T_{-e}=I$. If we have $f \in \mathfrak{M}_{s}(s=1,2, \cdots) f \neq 0$ and $H_{-} f=0$, then $0=H_{+} H_{-} f=(s(s-1)+q) f$, or $q=-s(s-1)$; the same is the situation if $f \in \mathfrak{M}_{-8}, f \neq 0$ and $H_{+} f=0$. Therefore, if $q>0$, then $\mathfrak{M}_{0} \neq(0)$. Let $e^{(i)}(i=1,2, \cdots, m ; 1 \leqq m \leqq+\infty)$ be a complete orthonormal system in it. We put

$$
\begin{array}{ll}
f_{s}^{(i)}=\left(H_{+}\right)^{s} e^{(i)}, & \\
f_{0}^{(i)}=e^{(i)}, & (s=1,2, \cdots ; i=1,2, \cdots, m) . \\
f_{-s}^{(i)}=\left(H_{-}\right)^{s} e^{(i)} &
\end{array}
$$


Then we have $\left(f_{s}^{(i)}, f_{t}^{(j)}\right)=0$ if $i \neq j$ or $s \neq t$. Denoting the subspace of $H$, spanned by the sequence $\left\{f_{s}^{(i)}\right\}$ with a fixed $i$, by $\mathfrak{N}_{i}$, we have $H=\sum_{i=1}^{m} \oplus \mathfrak{N}_{i}$, and the part of $T_{a}$ in any of these subspaces is an irreducible representation with $Q=q I$. But combined with the assumption that $T_{-e}=I$, this characterizes a representation of type $C_{q}^{0}$ uniquely (cf. end of $\S \mathrm{A}$ ). If $q<0$, then $\mathfrak{M}_{0}=(0)$, because if $H_{0} f=0$ and $f \neq 0$, then $H_{-} H_{+} f=q f$, or $\left\|H_{+} f\right\|^{2}=q(f, f)$, which implies $q \geqq 0$. Let $t$ be the smallest positive integer such that at least one of the equations $H_{0} f=t f, H_{0} f=-t f$ possesses nontrivial solution. In both cases we get $q=t(1-t)$. Suppose first that $\mathfrak{M}_{t} \neq(0)$. Let $e^{(i)}$ be a complete orthonormal system in $\mathfrak{M}_{t}$. We form the sequence

$$
f_{s}^{(i)}=\left(H_{+}\right)^{s-t} e^{(i)} \quad(s=t, t+1, \cdots ; i=1,2, \cdots, m) .
$$

Since $\left\|f_{s+1}^{(i)}\right\|^{2}=\left(H_{-} H_{+} f_{s}^{(i)}, f_{s}^{(i)}\right)=\left.(n(n+1)-t(t-1))\left\|f_{s}^{(i)}\right\|\right|^{2}, f_{s}^{(i)}$ never vanishes. Putting $\mathfrak{N}_{i}$ for the subspace spanned by the vectors $\left\{f_{s}^{(i)} ; s \geqq t\right\}$, one has $\sum_{i=1}^{m} \oplus \mathfrak{N}_{i}=\sum_{u \geqq t} \oplus \mathfrak{M}_{u}$. Moreover, one sees at once that each $\mathfrak{N}_{i}$ is invariant and irreducible under the joint action of the operators $H_{0}, H_{+}, H_{-}$, hence it is an irreducibly invariant subspace under $T_{a}$. Since the range of the spectrum of $H_{0}$ in any of the subspaces is $m=t, t+1, \cdots$, the part of $T_{a}$ in $\mathfrak{N}_{i}$ is of the type $D_{t}^{+}$(cf. $\left.\S \mathrm{A}\right)$. Similarly, one can show that the part of $T_{a}$ in $\sum_{u \geqq t} \oplus \mathfrak{M}_{-u}$ is a multiple of the representation $D_{t}^{-}$. This proves our statement for the case $q<0$. Finally, if $q=0$, then the part of $T_{a}$ in $\mathfrak{M}_{0}$ is the trivial representation, and one easily proves, by aid of the reasoning just applied, that the part of $T_{a}$ in its orthogonal complement is a multiple of $D_{1}^{+}$and $D_{1}^{-}$. The case when $T_{-e}=-I$ can be treated in an analogous manner. Finally, in the general case, one applies the reduction mentioned at the end of the previous section.

B3. We formulate the following Theorem I in a more general form than needed in the future, since its proof is essentially the same.

THEOREM I. Let $T_{a}$ be a continuous unitary representation of $\subseteq$ such that $T_{-\bullet}=I$ or $T_{-e}=-I$, and $Q$ its Casimir operator. Denoting the ring of all bounded functions of $Q$ by $R$, there exists a sequence $\mathfrak{M}_{n}(n=0,1,2, \cdots)$ of pairwise orthogonal subspaces the projections of which are in $R$ and whose sum is the whole space, with the following property. If $T_{a}^{(n)}$ denotes the part of $T_{a}$ in $\mathfrak{M}_{n}(n=0,1, \cdots)$, then $T_{a}^{(0)}$ is a discrete direct sum of representations of the discrete series and of the trivial representation; $T_{a}^{(n)}$ is an $n$-fold copy of a representation $S_{a}^{(n)}$, such that, denoting the subspace and Casimir operator corresponding to the latter by $H$ and $Q$ resp., we have

$$
H=\int \oplus H_{\lambda}(d \sigma)^{1 / 2}, \quad S_{a}^{(n)}=\sum S_{a}(\lambda),
$$

where $S_{a}(\lambda)$ is a continuous irreducible representation in $H_{\lambda}$ from one of the two continuous series, and in addition, $Q=\sum Q(\lambda)=\sum \lambda I$. 
For the proof we again take the case $T_{-e}=I$ first. We can write $Q$ in the form $Q_{0}+\sum_{\nu=1}^{\infty} \lambda_{\nu} Q_{\nu}$, such that the $Q_{\nu}$ are pairwise orthogonal projections in $R, Q_{0}$ does not possess nontrivial eigenelements, and $Q_{0} Q_{\nu}=0(\nu=1,2, \cdots)$. Now take those from among the projections $Q_{\nu}$, for which $\lambda_{\nu}$ is of the form $s(1-s)(s=1,2, \cdots)$, and denote their sum by $P_{0}$. Then we may conclude by aid of what was proved in B2 that the part of $T_{a}$ in the corresponding subspace is a discrete direct sum of representations of the discrete class and of the trivial representation. If $\lambda_{\nu}$ is not of this form, then, again by B2, it is $>0$, and the part of $T_{a}$ in the corresponding subspace is a multiple of a representation of the type $C_{\lambda_{\nu}}^{0}$. On the other hand, by $\S 1$ we can find a sequence of pairwise orthogonal projections $P_{n}(n=1,2, \cdots)$ in $R$, the sum of which is orthogonal to $\sum_{\nu} Q_{\nu}$, such that the part of $T_{a}$ in the subspace corresponding to $P_{n}$ is a continuous direct sum of unitary representations, each of which is an $n$-fold copy of an irreducible representation from one of the two continuous series. But adding to $P_{n}$ those $Q_{\nu}$, with $\lambda_{\nu}>0$, which are sums of $n$ irreducible projections, the new projection obtained in this way obviously possesses the properties claimed for $P_{n}$ above; we retain for it the same notation. To describe the situation in detail, we denote the subspace corresponding to $P_{n}$, the part of $T_{a}$ in it and its Casimir operator by $\mathfrak{M}_{n}$, $T_{a}^{(n)}$ and $Q_{n}$ resp. We may put

$$
\mathfrak{M}_{n}=\int \oplus H_{\lambda}(d \sigma)^{1 / 2} \text { and } T_{a}^{(n)}=\sum T_{a}^{(n)}(\lambda)
$$

such that $Q_{n}=\sum Q_{n}(\lambda)=\sum \lambda I$. What concerns $T_{a}^{(\boldsymbol{x})}(\lambda)$, we have a system $e_{s}^{(i)}(\lambda)(i=1,2, \cdots, n ; s=0, \pm 1, \pm 2, \cdots)$ of vector functions, such that for each fixed $\lambda$ the corresponding sequence of vectors forms a complete orthonormal system in $H_{\lambda}$, and, denoting the subspace spanned by the sequence $e_{s}^{(i)}(\lambda)$ for a fixed $i$ by $\mathfrak{M}_{\lambda}^{(i)}$, and the part of $T_{a}^{(n)}(\lambda)$ in it by $S_{a}^{(i)}(\lambda)$, the latter is an irreducible representation with a Casimir operator $Q^{(i)}(\lambda)$ $=\sum \lambda I$. Defining for $i \neq j$ a unitary mapping $U$ from $\mathfrak{M}_{\lambda}^{(i)}$ onto $\mathfrak{M}_{\lambda}^{(j)}$ by $U e_{s}^{(i)}(\lambda)=e_{s}^{(j)}(\lambda)$, then evidently $S_{a}^{(i)}(\lambda)$ goes over into $S_{a}^{(j)}(\lambda)$. Putting finally

$$
\mathfrak{M}^{(i)}=\int \oplus \mathfrak{M}_{\lambda}^{(i)}(d \sigma)^{1 / 2} \quad(i=1,2, \cdots, n),
$$

we have $\mathfrak{M}_{n}=\sum_{i=1}^{n} \mathfrak{M}^{(i)}$. The parts of $T_{a}^{(n)}$ in any two of these subspaces are unitary equivalent, and any of them, along with the corresponding subspace possesses the properties claimed for $S_{a}^{(n)}$ and $H$ in the formulation of Theorem I. Of course, we have $\sum_{n=0}^{\infty} P_{n}=I$.

The proof for the case $T_{-e}=-I$ is similar, and details will be omitted.

As we shall see later (cf. also Introduction), in the decomposition of the Kronecker product of two irreducible representations of $\mathfrak{S}$ either no representation from the principal continuous series occurs, or every representation from the principal continuous series occurs with the same multiplicity, and a 
weight function, equivalent to the Lebesgue measure. The representations of the type $E_{q}$ appear, similarly to the representations of the discrete series, as discrete direct summands and, as one can see from the classification in $\S \mathrm{A}$, they occur in the case $T_{-e}=I$ only.

In the following $\mathfrak{M}_{0}$ and $\mathfrak{M}_{1 / 2}$ will have the same meaning as in (1) and (2). The purpose of Corollaries A and B is to give an indication for the effective determination of the irreducible components of Kronecker products. The reason for their being formulated in the following way was given above.

Corollary A. Let $T_{a}$ be a unitary representation of $\mathfrak{S}$ such that $T_{-e}=I$, and $Q$ its Casimir operator. Suppose that the part $Q_{0}$ of $Q$ in $\mathfrak{M}_{0}$ is a direct sum of an operator $\bar{Q}_{0}$, which is an $n$-fold copy of an operator with a simple spectrum, and of an operator of the form

$$
\sum_{\nu=1}^{m} \lambda_{\nu} P_{\nu} \quad\left(m=1,2, \cdots, \infty ; \lambda, \geqq 0 ; \lambda_{\nu} \neq \lambda_{\mu} \text { for } \nu \neq \mu\right) .
$$

Then $T_{a}$ is a direct sum of two representations $T_{a}^{c}$ and $T_{a}^{d} . T_{a}^{c}$ is again a direct sum of two representations. The first is an $n$-fold copy of a representation, which is a continuous direct sum of irreducible representations $T_{a}(\lambda)$ of type $E_{\lambda}$ if $0<\lambda<1 / 4$ and $C_{\lambda}^{0}$ if $1 / 4 \leqq \lambda$, with respect to a weight function, equivalent to the spectral resolution of $\bar{Q}_{0}$. The second is a discrete direct sum of $\operatorname{dim} P_{\nu}$-fold copies of irreducible representations of type $E_{\lambda_{\nu}}$ if $0<\lambda_{\nu}<1 / 4$ or $C_{\lambda_{\nu}}^{0}$ if $\lambda_{\nu} \geqq 1 / 4$. $T_{a}^{d}$ is a discrete direct sum of representations of the discrete series and of the trivial representation. The number of times $D_{s}^{+}\left(D_{s}^{-}\right)$occurs in $T_{a}^{d}$ equals the dimension of the subspace

$$
\left\{f ; H_{-} f=0, H_{0} f=s f\right\} \quad\left(\left\{f ; H_{+} f=0, H_{0} f=-s f\right\} \text { resp. }\right) \quad(s=1,2, \cdots) .
$$

CoROllaRy B. Let $T_{a}$ be a unitary representation of $\subseteq$ such that $T_{-e}=-I$, and $Q$ its Casimir operator. Suppose that the part $Q_{0}$ of $Q$ in the subspace of $\mathfrak{M}_{1 / 2}$ orthogonal to the closed subspace $\left\{f ; f \in \mathfrak{M}_{1 / 2}, H_{-} f=0\right\}$ is an $n$-fold copy of an operator with a simple spectrum. Then $T_{a}$ is a direct sum of two representations $T_{a}^{c}$ and $T_{a}^{d} \cdot T_{a}^{c}$ is an $n$-fold copy of a representation, which is a continuous direct sum of irreducible representations $T_{a}(\lambda)$ of type $C_{\lambda}^{1 / 2}(\lambda>1 / 4)$ with respect to a weight function, equivalent to the spectral resolution of $Q_{0}$ in the interval $[1 / 4, \infty] . T_{a}^{d}$ is a discrete direct sum of representations of the discrete series and of the trivial representation. The number of times $D_{s}^{+}\left(D_{s}^{-}\right)$occurs in $T_{a}^{d}$ equals the dimension of the subspace

$\left\{f ; H_{-} f=0, H_{0} f=s f\right\} \quad\left(\left\{f ; H_{+} f=0, H_{0} f=-s f\right\}\right.$ resp. $) \quad(s=1 / 2,3 / 2, \cdots)$.

The proof for both corollaries can easily be put together from the considerations of $\S \S \mathrm{B} 1-\mathrm{B} 3)$.

C. Kronecker products of irreducible representations. For a theory of 
tensorial (Kronecker) products of Hilbert spaces we refer the reader to [8, Chapter II]. Here we restrict ourselves to a few simple remarks which will be useful in the following.

C1. Suppose that $A_{j}$ is a self-adjoint operator on the Hilbert space $H_{j}(j=1,2)$ resp. We put $U_{t}^{(j)}=\exp \left(-i A_{j} t\right)$. We form the Hilbert space $H=H_{1} \otimes H_{2}$ and define $U_{t}=U_{t}^{(1)} \otimes U_{i}^{(2)} \quad(-\infty<t<+\infty)$. Suppose that $U_{t}=\exp (-i A t)$. If $f_{j} \in D_{A_{j}}(j=1,2)$ resp., then $f_{1} \otimes f_{2} \in D_{A}$ and we have $A\left(f_{1}\right.$ $\left.\otimes f_{2}\right)=A_{1} f_{1} \otimes f_{2}+f_{1} \otimes A_{2} f_{2}$.

For the proof we recall that for any one-parameter group $U_{t}=\exp (-i A t)$ on a Hilbert space $H$, we have

$$
\lim _{t \rightarrow 0} \frac{1}{t}\left(U_{t}-I\right) f=-i A f \text { strongly, } \quad \text { if } f \in D_{A},
$$

and conversely, if the limits on the left-hand side exist, then $f \in D_{\boldsymbol{A}}$, and it equals $-i A f$. But

$$
\begin{aligned}
& \left\|\frac{1}{t} U_{t}\left(f_{1} \otimes f_{2}\right)+i\left(A_{1} f_{1}\right) \otimes f_{2}+f_{1} \otimes i\left(A_{2} f_{2}\right)\right\| \\
& \leqq
\end{aligned} \begin{aligned}
& \frac{1}{t}\left(U_{t}^{(1)}-I\right) f_{1} \otimes U_{t}^{(2)} f_{2}+i\left(A_{1} f_{1}\right) \otimes f_{2} \| \\
& \quad+\left\|f_{1} \otimes \frac{1}{t}\left(U_{t}^{(2)}-I\right) f_{2}+f_{1} \otimes i A_{2} f_{2}\right\| \rightarrow 0 \quad \text { if } t \rightarrow 0
\end{aligned}
$$

from which our statement easily follows.

C2. Let $T_{a}^{\left(q_{1}\right)}$ and $T_{a}^{\left(q_{2}\right)}\left(0<q_{1}, q_{2}<+\infty\right)$ two irreducible representations of the type $C_{q}^{0}$ or $E_{q}$ in the Hilbert spaces $H_{q_{1}}$ and $H_{q_{2}}$ resp. We know (cf. $\left[1\right.$, p. 604]) that for any irreducible representation $T_{a}^{(a)}$ of the above classes there exists a complete orthonormal system $e_{j}(j=0, \pm 1, \pm 2, \cdots)$, such that

$$
\begin{aligned}
& H_{0} e_{j}=j e_{j} \\
& H_{+} e_{j}=(q+j(j+1))^{1 / 2} e_{j+1}, \\
& H_{-} e_{j}=(q+j(j-1))^{1 / 2} e_{j-1}
\end{aligned}
$$

(for the definition of $H_{0}, H_{+}$and $H_{-}$cf. $\S \mathrm{B}$ ). We denote the notions corresponding to $T_{a}^{\left(a_{i}\right)}$ by $H_{0}^{(i)}, H_{+}^{(i)}, H_{-}^{(i)}$ and $e_{j}^{(i)}$ resp. $(i=1,2)$. Now we form the unitary representation $T_{a}=T_{a}^{\left(a_{1}\right)} \otimes T_{a}^{\left(q_{2}\right)}$ on $H_{q_{1}} \otimes H_{q_{2}}$. Then the system $\left\{e_{j}^{(1)} \otimes e_{k}^{(2)}\right\}(j, k=0, \pm 1, \pm 2, \cdots)$ is a complete orthonormal system in $H_{q_{1}} \otimes H_{q_{2}}$; in particular, the system $\left\{e_{j}^{(1)} \otimes e_{-j}^{(2)}\right\}(j=0, \pm 1, \pm 2, \cdots)$ is complete orthonormal in $\mathfrak{M}_{0}$. From (1) we can conclude that 


$$
\begin{aligned}
& H_{0} f=H_{0}^{(1)} f+H_{0}^{(2)} f, \\
& H_{+} f=H_{+}^{(1)} f+H_{+}^{(2)} f, \\
& H_{-} f=H_{-}^{(1)} f+H_{-}^{(2)} f,
\end{aligned}
$$

where $H_{0}, H_{+}$and $H_{-}$correspond to $T_{a}, f$ is a finite linear combination of members of the system $\left\{e_{j}^{(1)} \otimes e_{k}^{(2)}\right\}$; finally, we simply wrote $H_{0}^{(1)}, H_{+}^{(1)}, H_{-}^{(1)}$ instead of $H_{0}^{(1)} \otimes I, H_{+}^{(1)} \otimes I, H_{-}^{(1)} \otimes I$, etc.

C3. Let $Q$ be the Casimir operator corresponding to $T_{a}$, and $Q_{0}$ its restriction to $\mathfrak{M}_{0}$. Moreover we put $f_{m}=e_{m}^{(1)} \otimes e_{-m}^{(2)}(m=0, \pm 1, \pm 2, \cdots)$. Next we assert that $Q_{0}$ is the closure of its restriction to the set $D$ of all finite linear combinations of the system $\left\{f_{m}\right\}$.

For the proof observe that, using relations $\left({ }^{*}\right)$ above, an easy calculation gives

$$
Q_{0} f_{m}=(1 / 2)\left(H_{+} H_{-}+H_{-} H_{+}\right) f_{m}=h_{m+1, m} f_{m+1}+h_{m, m} f_{m}+h_{m-1, m} f_{m-1},
$$

and $h_{m, n}=\overline{h_{n, m}}$. Hence $[1$, Lemma 3, p. 608] the closure of the restriction of $Q_{0}$ to $\mathfrak{M}_{0}$ is self-adjoint, which proves our statement.

An analogous result holds for the product of any two irreducible representations (if $T_{-e}=-I, \mathfrak{M}_{0}$ is to be replaced by $\mathfrak{M}_{1 / 2}$ ). But since its proof requires trivial modifications only, we omit it.

\section{Chapter II. Products of Representations of the CONTINUOUS SERIES OF $L_{3}$}

In what follows we shall investigate the decomposition of the product $T_{a}=T_{a}^{\left(a_{1}\right)} \otimes T_{a}^{\left(a_{2}\right)}(a \in \mathfrak{S})$, where the representations

$$
T_{a}^{\left(q_{1}\right)}, T_{a}^{\left(q_{2}\right)} \quad\left(0<q_{1}, q_{2}<+\infty\right)
$$

defined on the Hilbert spaces $H_{q_{1}}$ and $H_{q_{2}}$ resp. are from one of the series $C_{a}^{0}$ or $E_{q}$ (cf. I.A). We know that their Casimir operators equal $q_{1} I$ and $q_{2} I$ resp.

(A) It follows from Theorem I in I.B that our representation is a direct sum of two representations $T_{a}^{d}$ and $T_{a}^{c}$, made up of representations of the discrete and continuous series resp. $\left({ }^{7}\right)$. Now we are going to show that $T_{a}^{d}$ is a discrete direct sum of representations, containing exactly one summand from each of the classes $D_{s}^{+}, D_{s}^{-}(s=1,2, \cdots)$ resp. By Corollary A in I.B we have to show that for $f \in H_{q_{1}} \otimes H_{q_{2}}$ and a positive integer $s$ the equations $H_{0} f=s f$, $H_{-} f=0$, and $H_{0} f=-s f, H_{+} f=0$ have exactly one solution resp.

We recall that with the notations of I.C.2 we have $H_{0}=H_{0}^{(1)}+H_{0}^{(2)}$, $H_{+}=H_{+}^{(1)}+H_{+}^{(2)}, H_{-}=H_{-}^{(1)}+H_{-}^{(2)}$ on finite linear combinations of the system $\left\{e_{i}^{(1)} \otimes e_{j}^{(2)}\right\}$. In the following we deal with the case $s>0$ only; the opposite

(7) It will turn out later (cf. II.B.4) that $T_{a}$ does not contain the trivial representation. 
sign can be settled in an analogous way. It is clear that $H_{0} f=s f$ implies

$$
f=\sum_{j=-\infty}^{\infty} a_{j}\left(e_{j}^{(1)} \otimes e_{s-j}^{(2)}\right), \quad \sum_{j=-\infty}^{\infty}\left|a_{j}\right|^{2}<+\infty .
$$

Writing out the condition for $H_{-} f=0$ purely formally, we get

$$
\begin{aligned}
H_{-} f= & \sum_{j=-\infty}^{\infty} a_{j}\left(H_{-}^{(1)} e_{j}^{(1)} \otimes e_{s-j}^{(2)}\right)+\sum_{j=-\infty}^{\infty} a_{j}\left(e_{j}^{(1)} \otimes H_{-}^{(2)} e_{s-j}^{(2)}\right) \\
= & \sum_{j=-\infty}^{\infty} a_{j}\left(q_{1}+j(j-1)\right)^{1 / 2}\left(e_{j-1}^{(1)} \otimes e_{s-j}^{(2)}\right) \\
& +\sum_{j=-\infty}^{\infty} a_{j}\left(q_{2}+(j-s)(j+1-s)\right)^{1 / 2}\left(e_{j}^{(1)} \otimes e_{s-j-1}^{(2)}\right) \\
= & \sum_{j=-\infty}^{\infty}\left(a_{j+1}\left(q_{1}+j(j+1)\right)^{1 / 2}\right. \\
& \left.+a_{j}\left(q_{2}+(j-s)(j+1-s)\right)^{1 / 2}\right)\left(e_{j}^{(1)} \otimes e_{s-j-1}^{(2)}\right)=0 .
\end{aligned}
$$

This gives

$$
a_{j+1}\left(q_{1}+j(j+1)\right)^{1 / 2}+a_{j}\left(q_{2}+(j-s)(j+1-s)\right)^{1 / 2}=0 .
$$

Consequently, if $a_{0} \neq 0$, then $a_{j} \neq 0(j= \pm 1, \pm 2, \cdots)$. Furthermore, for $n=1,2, \cdots, a_{n+1}=\gamma_{n} a_{1}$, with

$$
\begin{aligned}
\gamma_{n} & =(-1)^{n}\left(\prod_{j=1}^{n}\left(\frac{q_{2}+(j+1-s)(j-s)}{q_{1}+j(j+1)}\right)^{1 / 2}\right) \\
& =(-1)^{n}\left(\prod_{j=1}^{n}\left(\frac{1-\frac{2 s}{j+1}+\frac{s^{2}-s+q_{2}}{j(j+1)}}{1+\frac{q_{1}}{j(j+1)}}\right)\right)^{1 / 2} .
\end{aligned}
$$

Hence $(-1)^{n} \gamma_{n} \sim C n^{-s}$, where $C$ does not depend on $n$. Similarly $a_{-n}=\delta_{n} a_{0}$ with

$$
\delta_{n}=(-1)^{n}\left(\prod_{j=0}^{n-1}\left(\frac{1+\frac{q_{1}}{j(j+1)}}{1+\frac{2 s}{j+1}+\frac{s^{2}+s+q_{2}}{j(j+1)}}\right)\right)^{1 / 2}(n=1,2, \cdots) .
$$

Hence $(-1)^{n} \delta_{n} \sim C_{1} n^{-s}, C_{1}$ not depending on $n$. So we have

$$
\sum_{j=-\infty}^{\infty}\left|a_{j}\right|^{2}<+\infty .
$$


Conversely, one easily verifies that the element

$$
f=\sum_{j=-\infty}^{\infty} a_{j}\left(e_{j}^{(1)} \otimes e_{s-j}^{(2)}\right)
$$

satisfies all of our requirements. We have already seen that it is uniquely determined by them.

We shall see later that $T_{a}^{\left(q_{1}\right)} \otimes T_{a}^{\left(q_{2}\right)}$ does not contain the trivial representation (cf. 4.a. $\beta$ in $\S \mathrm{B}$ ).

B. Our next objective is to investigate the spectrum of the restriction $Q_{0}$ of the Casimir operator $Q$ of $T_{a}^{\left(q_{1}\right)} \otimes T_{a}^{\left(a_{2}\right)}$ to $\mathfrak{M}_{0}$ (cf. Corollary A in I.B). In order to do this, first we represent $\mathfrak{M}_{0}$ as a certain Hilbert space of functions by aid of Bargmann's description of representations of the type $C_{q}^{0}$ and $E_{q}$. Then $Q_{0}$ will turn out to be a certain linear differential operator of second order. We know that through the spectral properties of $Q_{0}$ we shall be able to determine the part $T_{a}^{e}$ of $T_{a}^{\left(a_{1}\right)} \otimes T_{a}^{\left(q_{2}\right)}$, made up of representations of the continuous series.

B1. First we recall the description of the representations $T_{a}^{(q)}(0<q<+\infty)$ (cf. $[1, \S \S 6,8])$.

(a) For $q \geqq 1 / 4, T_{a}^{(a)} \in C_{q}^{0}$. Let $H_{q}$ be the Hilbert space of the squareintegrable functions, with respect to the Lebesgue measure, over the circumference $T^{1}$ of the unit circle, with a norm

$$
(f, f)=\frac{1}{2 \pi} \int_{0}^{2 \pi}|f(\phi)|^{2} d \phi .
$$

For

$$
a=\left(\begin{array}{ll}
\alpha & \beta \\
\bar{\beta} & \bar{\alpha}
\end{array}\right), \quad|\alpha|^{2}-|\beta|^{2}=1,
$$

we put $w(a, \phi)=\alpha+\beta e^{i \phi}$, and define $\phi^{\prime}=a \phi$ through the condition

$$
e^{i \phi^{\prime}}=e^{i \phi} \overline{w(a, \phi)} / w(a, \phi) .
$$

Then for $f \in H_{q} T_{a}^{(q)}$ is defined by

$$
\left(T_{a}^{(q)} f\right)(\phi)=[\mu(a, \phi)]^{1 / 2+\sigma} f\left(a^{-1} \phi\right)
$$

where $\sigma=(1 / 4-q)^{1 / 2}, \operatorname{Im} \sigma>0$ and $\mu(a, \phi)=|w(a, \phi)|^{2}$. Observe that if

$$
g_{0}(\tau)=\left(\begin{array}{ll}
e^{-i \tau / 2} & 0 \\
0 & e^{i \tau / 2}
\end{array}\right) \quad(0 \leqq \tau \leqq 2 \pi)
$$

then

$$
\left(T_{g_{0}(\tau)}^{(q)} f\right)(\phi)=f\left(\phi+^{-} \tau\right)
$$


(b) If $0<q<1 / 4, T_{a}^{(a)}$ is of the type $E_{q}$. The Hilbert space $H_{q}$ is defined as follows. For $\sigma=(1 / 4-q)^{1 / 2}>0$, we put

$$
K_{\sigma}(\phi)=2^{-\sigma / 2} \pi(B(\sigma, 1 / 2))^{-1}(1-\cos \phi)^{\sigma-1 / 2}
$$

where $B(x, y)$ is Euler's Beta function $\Gamma(x) \Gamma(y) / \Gamma(x+y)$. This function is in $L^{1}\left(T^{1}\right)$; moreover we have

$$
\frac{1}{2 \pi} \int_{0}^{2 \pi} K_{\sigma}(\phi) e^{-i m \phi} d \phi=\lambda_{m}(\sigma)
$$

where

$$
\lambda_{m}(\sigma)=\prod_{j=1}^{m}\left(\frac{j-1 / 2-\sigma}{j-1 / 2+\sigma}\right)<1
$$

and $\lambda_{0}(\sigma)=1, \lambda_{m}(\sigma)=\lambda_{-m}(\sigma)(m=1,2, \cdots)$. Hence for any square integrable function

$$
f(\phi) \sim \sum a_{m} e^{i m \phi}
$$

we have

$$
\begin{aligned}
(f, f)_{\sigma} & =\frac{1}{(2 \pi)^{2}} \int_{0}^{2 \pi} \int_{0}^{2 \pi} K_{\sigma}(\phi-\psi) f(\phi) \overline{f(\psi)} d \phi d \psi \\
& =\sum_{-\infty}^{\infty} \lambda_{m}(\sigma)\left|a_{m}\right|^{2}<+\infty
\end{aligned}
$$

We define $H_{q}$ as the completion of $L^{2}\left(T^{1}\right)$ with respect to this norm. For a sufficiently regular $f \in H_{q}$ we have again

$$
\left(T_{a}^{(q)} f\right)(\phi)=[\mu(a, \phi)]^{1 / 2+\sigma} f\left(a^{-1} \phi\right) .
$$

In particular

$$
\left(T_{g_{0}(\tau)}^{(q)} f\right)(\phi)=f(\phi+\tau) .
$$

In both of the cases (a) and (b) putting $-i H_{j}=\Lambda_{j}(j=0,1,2)$, the latter are differential operators, defined as follows

$$
\begin{aligned}
& \Lambda_{0}=-\frac{\partial}{\partial \phi}, \quad \Lambda_{1}=\cos \phi \frac{\partial}{\partial \phi}-(1 / 2+\sigma) \sin \phi, \\
& \Lambda_{2}=\sin \phi \frac{\partial}{\partial \phi}+(1 / 2+\sigma) \cos \phi .
\end{aligned}
$$

B2. (a) Now we are going to determine the space $\mathfrak{M}_{0} \subset H_{q_{1}} \otimes H_{q_{2}}$. Here we have to consider three cases. 
( $\alpha) q_{1}, q_{2} \geqq 1 / 4$. Then $H_{q_{1}} \otimes H_{q_{2}}$ is the space of square integrable functions over $T^{1} \times T^{1}$. Since for $f$ in this space and $a=g_{0}(\tau)$

$$
\left(T_{a}^{\left(q_{1}\right)} \otimes T_{a}^{\left(q_{2}\right)} f\right)\left(\phi_{1}, \phi_{2}\right)=f\left(\phi_{1}+\tau, \phi_{2}+\tau\right),
$$

$\mathfrak{M}_{0}$ is the subspace consisting of functions of the form $f\left(\phi_{1}-\phi_{2}\right)$. Of course, this is isomorphic to $L^{2}\left(T^{1}\right)$ in an obvious way. have

( $\beta) q_{1} \geqq 1 / 4,0<q_{2}<1 / 4$. If $f \in H_{q_{1}} \otimes H_{q_{2}}$ is sufficiently regular, then we

$$
\begin{array}{r}
\|f\|^{2}=\frac{1}{(2 \pi)^{3}} \int_{0}^{2 \pi} \int_{0}^{2 \pi} \int_{0}^{2 \pi} f\left(\phi_{1}, \phi_{2}^{\prime}\right) \overline{f\left(\phi_{1}, \phi_{2}^{\prime \prime}\right)} K_{\sigma}\left(\phi_{2}^{\prime}-\phi_{2}^{\prime \prime}\right) d \phi_{1} d \phi_{2}^{\prime} d \phi_{2}^{\prime \prime} \\
\left(\sigma=\left(1 / 4-q_{2}\right)^{1 / 2}>0\right) .
\end{array}
$$

Similarly, as in $(\alpha)$ one sees that if in addition $f \in \mathfrak{M}_{0}$, then it must again be of the form $f\left(\phi_{1}-\phi_{2}\right)$, and in this case we have

$$
\begin{aligned}
\|f\|^{2} & =\frac{1}{(2 \pi)^{3}} \int_{0}^{2 \pi} \int_{0}^{2 \pi} \int_{0}^{2 \pi} f\left(\phi_{1}-\phi_{2}^{\prime}\right) \overline{f\left(\phi_{1}-\phi_{2}^{\prime \prime}\right)} K_{\sigma}\left(\phi_{2}^{\prime}-\phi_{2}^{\prime \prime}\right) d \phi_{1} d \phi_{2}^{\prime} d \phi_{2}^{\prime \prime} \\
& =\frac{1}{(2 \pi)^{2}} \int_{0}^{2 \pi} \int_{0}^{2 \pi} f\left(\phi^{\prime}\right) \overline{f\left(\phi^{\prime \prime}\right)} K_{\sigma}\left(\phi^{\prime}-\phi^{\prime \prime}\right) d \phi^{\prime} d \phi^{\prime \prime} .
\end{aligned}
$$

Hence $\mathfrak{M}_{0}$ can be identified with $H_{q_{2}}$ in a natural way.

( $\gamma$ ) $0<q_{1}, q_{2}<1 / 4$. Proceeding as before, $f \in \mathfrak{M}_{0}$ is again of the form $f\left(\phi_{1}-\phi_{2}\right)$. Putting $\sigma=\left(1 / 4-q_{1}\right)^{1 / 2}, \tau=\left(1 / 4-q_{2}\right)^{1 / 2}$, we have

$$
\begin{aligned}
&\|f\|^{2}=\frac{1}{(2 \pi)^{2}} \int_{0}^{2 \pi} \int_{0}^{2 \pi} \int_{0}^{2 \pi} \int_{0}^{2 \pi} f\left(\phi_{1}-\phi_{2}^{\prime}\right) f\left(\phi_{1}-\phi_{2}^{\prime \prime}\right) K_{\sigma}\left(\phi_{1}-\phi_{1}^{\prime}\right) K_{\tau}\left(\phi_{2}-\phi_{2}^{\prime}\right) \\
& \cdot d \phi_{1} d \phi_{2} d \phi_{1}^{\prime} d \phi_{2}^{\prime}
\end{aligned}
$$

where $K_{\sigma, \tau}(\phi)$ is $1 / 2 \pi$ times the convolution of the two integrable functions $K_{\sigma}(\phi)$ and $K_{\tau}(\phi)$. We denote the completion of this by $H_{\sigma, \tau}$. For convenience we extend this definition as follows. We define $H_{\sigma, \tau}$ as $L_{2}\left(T^{1} \times T^{1}\right)$ if $\sigma$ and $\tau$ are both purely imaginary or zero, and $H_{q}$ if $\sigma$ is purely imaginary or zero, and $\tau=(1 / 4-q)^{1 / 2}(0<q<1 / 4)$, or conversely. Then summing up, in all cases $\mathfrak{M}_{0}$ can be identified with $H_{\sigma, \tau}$ in a natural way if $\sigma=\left(1 / 4-q_{1}\right)^{1 / 2}$ and $\tau=\left(1 / 4-q_{2}\right)^{1 / 2}$ (the sign of the square-root has already been disposed of in each case). Finally, putting $\lambda_{m}(\sigma) \equiv 1(m=0, \pm 1, \pm 2, \cdots)$ for $\sigma$ purely imaginary or zero, and $\lambda_{\sigma, \tau}(m)=\lambda_{m}(\sigma) \lambda_{m}(\tau)(m=0, \pm 1, \pm 2, \cdots)$ for $\sigma, \tau$ 
either purely imaginary, zero, or between 0 and $1 / 2$, the system of functions

$$
e_{m}(\phi)=\left(\lambda_{\sigma, \tau}(m)\right)^{-1 / 2} e^{i m \phi}
$$

forms a complete orthonormal system in $H_{\sigma, \tau}$. For $f, g \in H_{\sigma, \tau}$ we denote the inner product by $(f, g)_{\sigma, \tau}$.

(b) Next we compute the Casimir operator $Q$ of $T_{a}^{\left(a_{1}\right)} \otimes T_{a}^{\left(a_{2}\right)}$ and its restriction $Q_{0}$ to $\mathfrak{M}_{0}$. Let $f\left(\phi_{1}, \phi_{2}\right)$ be a sufficiently regular function in $\mathfrak{M}_{0}$, and $\Lambda_{j}^{(1)}$ and $\Lambda_{j}^{(2)}(j=0,1,2)$ the operators, described in B1, acting on $\phi_{1}$ and $\phi_{2}$ resp. Then for any such $f$

$$
Q=\left(\Lambda_{0}^{(1)}+\Lambda_{0}^{(2)}\right)^{2}-\left(\Lambda_{1}^{(1)}+\Lambda_{1}^{(2)}\right)^{2}-\left(\Lambda_{2}^{(1)}+\Lambda_{2}^{(2)}\right)^{2} .
$$

Inserting the expressions for $\Lambda_{j}^{(i)}(i=1,2 ; j=0,1,2)$, and putting $A=\sigma+1 / 2, B=\tau+1 / 2\left(\sigma=\left(1 / 4-q_{1}\right)^{1 / 2}, \tau=\left(1 / 4-q_{2}\right)^{1 / 2}\right)$, we get

$$
\begin{aligned}
Q=q_{1}+q_{2}+2\left\{\left(1-\cos \left(\phi_{1}-\phi_{2}\right)\right) \frac{\partial^{2}}{\partial \phi_{1} \partial \phi_{2}}-B \sin \left(\phi_{1}-\phi_{2}\right) \frac{\partial}{\partial \phi_{1}}\right. \\
\left.+A \sin \left(\phi_{1}-\phi_{2}\right) \frac{\partial}{\partial \phi_{2}}-A B \cos \left(\phi_{1}-\phi_{2}\right)\right\} .
\end{aligned}
$$

Putting for reasons of convenience $L=-Q_{0} / 2$, we have for a sufficiently regular $f \in H_{\sigma, \tau}$

$$
L f=(1-\cos \phi) f^{\prime \prime}+(A+B) \sin \phi f^{\prime}+\left(A B \cos \phi-\left(q_{1}+q_{2}\right) / 2\right) f .
$$

Furthermore, for $f(\phi)=(1-\cos \phi)^{-(\sigma+\tau) / 2} v(\phi)$

$$
L(1-\cos \phi)^{-(\sigma+\tau) / 2} v(\phi) \equiv(1-\cos \phi)^{-(\sigma+\tau) / 2} L^{0} v(\phi)
$$

where

$$
L^{0} v(\phi)=\frac{d}{d \phi}(1-\cos \phi) \frac{d v}{d \phi}+\left(\frac{1}{4}(A-B)^{2}-\frac{1}{4}\right)(1-\cos \phi) v \quad(0 \leqq \phi \leqq 2 \pi) .
$$

Finally, observe that as a consequence of I.C.3 $Q_{0}$ is the closure of its restriction to the sufficiently regular elements of $H_{\sigma, \tau}$.

B3. In order to investigate the spectral properties of $Q_{0}$, we are going to determine the operator $\left(Q_{0}-\lambda I\right)^{-1}=R(\lambda)$ in $H_{\sigma \tau}$ for $\operatorname{Im} \lambda \neq 0$. First of all, we determine the solutions of $L^{0} f-\lambda f=0$. Making the substitution $\tau=\sin ^{2} \phi / 2$ $(0 \leqq \phi \leqq \pi)$ in this equation, it goes over into

$$
\tau(1-\tau) \frac{d^{2} f}{d \tau^{2}}+\left(\frac{3}{2}-2 \tau\right) \frac{d f}{d t}-\left(\frac{1}{4}-\left(\frac{A-B}{2}\right)^{2}+\frac{\lambda}{2 \tau}\right) f=0 .
$$

Performing the further substitution $f(\tau)=\tau^{p} g(\tau)$ with $p=-1 / 4+$ $(1 / 4+2 \lambda)^{1 / 2} / 2$, we see that $g(\tau)$ satisfies the hypergeometric equation 


$$
\tau(1-\tau) \frac{d^{2} g}{d \tau^{2}}+(c-(a+b+1) \tau) \frac{d g}{d t}-a b g=0
$$

with

$$
a=1 / 4+(\sigma-\tau) / 2+\mu / 2, \quad b=1 / 4-(\sigma-\tau) / 2+\mu / 2, \quad c=1+\mu
$$

where $\mu=(1 / 4+2 \lambda)^{1 / 2}$. What concerns $\mu$, we shall specify the value of the square root later.

As it is well known (cf. [2, p. 247]), the two linearly independent solutions for $\left(^{*}\right)$ are $(0 \leqq \tau \leqq 1)$

$$
\begin{aligned}
& y_{1}(\tau)=F(a, b, c, \tau), \\
& y_{2}(\tau)=\tau^{1-c} F(1+a-c, 1+b-c, 2-c, \tau) .
\end{aligned}
$$

Since $\sin ^{2} \phi / 2=(1-\cos \phi) / 2$, the two independent solutions for the equation $L_{0} f-\lambda f=0$ in the interval $0<\phi \leqq \pi$ are given by

$$
\begin{aligned}
& f_{1}(\phi, \lambda)=(1-\cos \phi)^{-1 / 4+\mu / 2} F\left(a, b, c, \sin ^{2} \phi / 2\right), \\
& f_{2}(\phi, \lambda)=(1-\cos \phi)^{-1 / 4-\mu / 2} F\left(1+a-c, 1+b-c, 2-c, \sin ^{2} \phi / 2\right.
\end{aligned}
$$

with the values of $a, b, c$ given above.

Putting $f^{T}(\phi)=f(2 \pi-\phi)(0 \leqq \phi \leqq 2 \pi)$, one sees at once that $\left(L^{0} f\right)^{T}=L^{0}\left(f^{T}\right)$; therefore $f_{1}$ and $f_{2}$ give two independent solutions for $L^{0} f-\lambda f=0$ in the interval $\pi \leqq \phi<2 \pi$ too. So finally we can determine two independent solutions $g_{1}(\phi, \lambda)$ and $g_{2}(\phi, \lambda)$ of $L^{0} f-\lambda f=0$ in the interval $(0,2 \pi)$, such that $g_{1}(\phi, \lambda) \equiv$ $f_{1}(\phi, \lambda)$ for $0<\phi \leqq \pi$, and $g_{2}(\phi, \lambda) \equiv\left(g_{1}(\phi, \lambda)\right)^{T} \equiv g_{1}(2 \pi-\phi, \lambda)$ if $0<\phi<2 \pi$.

In the following we denote the class of functions on the circumference of the unit circle, indefinitely differentiable and vanishing in a neighborhood of 0 , by $C$. As it is known, for $f \in C$ the expression

$$
\begin{aligned}
F(\phi, \lambda)= & 1 / D(\lambda)\left(g_{2}(\phi, \lambda) \int_{0}^{\phi} f(\psi) g_{1}(\psi, \lambda) d \psi\right. \\
& \left.+g_{1}(\phi, \lambda) \int_{\phi}^{2 \pi} f(\psi) g_{2}(\psi, \lambda) d \psi\right) \quad(0<\phi<2 \pi)
\end{aligned}
$$

gives a solution of the equation $L^{0} F-\lambda F=f$, where

$$
D(\lambda)=2\left|\begin{array}{ll}
g_{1}(\pi, \lambda) & g_{2}(\pi, \lambda) \\
g_{1}^{\prime}(\pi, \lambda) & g_{2}^{\prime}(\pi, \lambda)
\end{array}\right|=-4 g_{1}(\pi, \lambda) g_{1}^{\prime}(\pi, \lambda),
$$

taking in view that $g_{1}(\pi, \lambda)=g_{2}(\pi, \lambda)$, and $g_{1}^{\prime}(\pi, \lambda)=-g_{2}^{\prime}(\pi, \lambda)$.

Observing that, putting $a(\phi)=(1-\cos \phi)^{-(\sigma+\tau) / 2}$, we have for $f \in C Q_{0} a(\phi) f$ $=-2 a(\phi) L^{0} f$, it is clear that 


$$
\begin{aligned}
\Psi_{f}(\phi, \lambda)= & a(\phi) / D(\lambda)\left(g_{2}(\phi, \lambda) \int_{0}^{\phi} g_{1}(\psi, \lambda)(a(\psi))^{-1} f(\psi) d \psi\right. \\
& \left.+g_{1}(\phi, \lambda) \int_{0}^{2 \pi} g_{2}(\psi, \lambda)(a(\psi))^{-1} f(\psi) d \psi\right)
\end{aligned}
$$

satisfies $Q_{0} \Psi_{f}-\lambda \Psi_{f}=f$, provided that we replace $2 \lambda$ by $-\lambda$ in $\mu$ occurring in $g_{1}$ and $g_{2}$. Concerning the function $\mu$ we make the following choice. Let $Z_{0}$ be that domain of the complex plane which is the complement of the halfline $1 / 4-i t(t \geqq 0)$. In the future $\mu$ will denote that branch of $(1 / 4-\lambda)^{1 / 2}$, which is positive for $\lambda<1 / 4$. It is singlevalued and regular everywhere within $Z_{0}$. For $\operatorname{Im} \lambda>0$ we have $\operatorname{Re} \mu>0$.

It is clear that if $\lambda$ is chosen in such a way in the upper half-plane that $\operatorname{Re} \mu$ is sufficiently large, then $\Psi_{f}(\phi, \lambda) \in H_{\sigma, \tau}$ and thus we may write $\Psi_{f}=$ $R(\lambda) f$, where $R(\lambda)=(Q-\lambda I)^{-1}\left({ }^{8}\right)$.

Before proceeding with the analysis of the operator $Q_{0}$, we compute $g_{1}(\pi, \lambda)$ and $g_{1}^{\prime}(\pi, \lambda)$. For this we recall the following three relations concerning the hypergeometric function (cf. [2, p. 251, and p. 267 , example 8, p. 248 , example 2]).

( $\alpha)$ If $\operatorname{Re}(c-a-b)>0$ then

$$
F(a, b, c, 1)=\frac{\Gamma(c) \Gamma(c-a-b)}{\Gamma(c-a) \Gamma(c-b)} .
$$

( $\beta)$ If $\operatorname{Re}(c-a \dot{-} b)<0$ then

$$
\begin{aligned}
& \lim _{\tau \rightarrow 1-0} \frac{F(a, b, c, \tau)}{(1-\tau)^{c-a-b}}=\frac{\Gamma(c) \Gamma(a+b-c)}{\Gamma(a) \Gamma(b)} . \\
& (\gamma) \frac{d}{d \tau} F(a, b, c, \tau)=\frac{a b}{c} F(a+1, b+1, c+1, \tau) .
\end{aligned}
$$

Since in our case $c-a-b=1 / 2$, and

$$
g_{1}(\phi, \lambda)=(1-\cos \phi)^{-1 / 4+\mu / 2} F\left(a, b, c, \sin ^{2} \phi / 2\right) \quad(0<\phi \leqq \pi)
$$

we have

$$
\begin{aligned}
g_{1}(\pi, \lambda) & =2^{-1 / 4+\mu / 2} F(a, b, c, 1) \\
& =2^{-1 / 4+\mu / 2} \frac{\Gamma(1+\mu) \Gamma(1 / 2)}{\Gamma(3 / 4+(\sigma-\tau) / 2+\mu / 2) \Gamma(3 / 4-(\sigma-\tau) / 2+\mu / 2)}
\end{aligned}
$$

and

(8) With a $\operatorname{Re} \mu$ sufficiently large $\Psi_{f}(\phi, \lambda) \in L^{2}\left(T^{1}\right)$, and the latter can be identified with a dense subset of $H_{\sigma, r}$. 


$$
\begin{aligned}
g_{1}^{\prime}(\pi, \lambda)= & \lim _{\phi \rightarrow \pi-0}(\mu / 2-1 / 4) \sin \phi(1-\cos \phi)^{-1 / 4+\mu / 2} F\left(a, b, c, \sin ^{2} \phi / 2\right) \\
& +2^{-1 / 4+\mu / 2} \lim _{\phi \rightarrow \pi-0}(\sin \phi / 2)(\cos \phi / 2) F^{\prime}\left(a, b, c, \sin ^{2} \phi / 2\right) \\
= & 2^{-1 / 4+\mu / 2} \lim _{\tau \rightarrow 1-0}(1-\tau)^{1 / 2} \frac{a b}{c} F(a+1, b+1, c+1, \tau) \\
= & 2^{-1 / 4+\mu / 2} \frac{\Gamma(1+\mu) \Gamma(1 / 2)}{\Gamma(1 / 4+(\sigma-\tau) / 2+\mu / 2) \Gamma(1 / 4-(\sigma-\tau) / 2+\mu / 2)} .
\end{aligned}
$$

Observe that both $g_{1}(\pi, \lambda)$ and $g_{1}^{\prime}(\pi, \lambda)$, and hence $D(\lambda)$ are regular functions of $\lambda$ in $Z_{0}$, since in that case $1+\mu \neq 0$.

B4. In the following we denote the subspaces of $H_{\sigma, \tau}$ generated by the systems of functions $\{\cos n \phi\}$ and $\{\sin n \phi\}(n=0,1,2, \cdots)$ by $H_{\sigma, \tau}^{+}$and $H_{\sigma, \tau}^{-}$resp. It is clear that they are orthogonal to each other and $H_{\sigma, \tau}=H_{\sigma, \tau}^{+} \oplus H_{\sigma, \tau}^{-}$. Moreover, each of them is left invariant by $Q_{0}$. We denote the part of $Q_{0}$ in $H_{\sigma, \tau}^{+}$and $H_{\sigma, \tau}^{-}$by $Q_{0}^{+}$and $Q_{0}^{-}$resp. Our chief objective in this section will be to investigate their spectrum, which will solve the question of the irreducible representations of the two continuous series occurring among the irreducible components of our Kronecker product (cf. Theorem and Corollary A in I.B). It turns out that the spectrum of both are simple and fill out the interval $[1 / 4, \infty]$, where they are equivalent to the Lebesgue measure. If in addition $\operatorname{Re}(\sigma+\tau)>1 / 2$ (in which case, of course, $\sigma$ and $\tau$ both are real), $Q_{0}^{+}$possesses an eigenvalue $\lambda_{0}=\left(1-(\sigma+\tau-1 / 2)^{2}\right) / 4,0<\lambda_{0}<1 / 4$. In other words, $Q_{0}$ is the direct sum of a two-fold copy of an operator with an absolutely continuous spectrum, and possibly of a scalar multiple of some one-dimensional projection.

(a) Case of $H_{\sigma, \tau}^{+}$. ( $\left.\alpha\right)$ To prove that the spectrum of $Q_{0}^{+}$is simple, it is enough to show that the linear span of the sequence $\left(Q_{0}\right)^{n} f_{0}(n=0,1, \cdots)$, where $f_{0}(\phi) \equiv 1$, contains the system $\{\cos n \phi\}$. For this it is obviously enough to show that $Q \cos \nu \phi$ is an even trigonometrical polynomial of degree $\nu+1$. For $\nu=0$ the statement is trivial. We assume it true for $0 \leqq \nu \leqq n-1(n>1)$. It is clear that $Q \cos n \phi$ is at most of degree $n+1$; but an elementary computation shows that the coefficient of $\cos (n+1) \phi$ is $-(n+1 / 2+\sigma) \times$ $(n+1 / 2+\tau) / 4 \neq 0$, which proves our statement.

( $\beta$ ) Next we discuss the part of the spectrum of $Q_{0}^{+}$in $(-\infty, 1 / 4)$. As in B3 we put again $R(\lambda)=\left(Q_{0}-\lambda I\right)^{-1}$ for $\operatorname{Im} \lambda \neq 0$. If $f \in C, f^{T}=f$, then $f \in H_{\sigma, \tau}^{+}$, and $(R(\lambda) f, f)_{\sigma, \tau} \equiv h_{f}(\lambda)$ is an analytic function of $\lambda$ for $\operatorname{Im} \lambda \neq 0$. Moreover, since $Q_{0}^{+} \geqq 0$, this is true even for $\lambda<0$. Now we are going to prove that $h_{f}(\lambda)$ possesses a continuation over the segment $[0,1 / 4)$, which is regular except possibly for a pole at $0<\lambda_{0}<1 / 4$, if $\operatorname{Re}(\sigma+\tau)>1 / 2$. We know that for $\operatorname{Re} \mu$ sufficiently large $R(\lambda) f=\Psi_{f}(\phi, \lambda)$, where $\Psi_{f}(\phi, \lambda)$ is defined by expression (*) in B3. We have, along with $f,\left[\Psi_{f}(\phi, \lambda)\right]^{T} \equiv \Psi_{f}(\phi, \lambda)$. Next we form the Fourier coefficients $a_{n}(\lambda)$ of $\Psi_{f}(\phi, \lambda)$ ( $f$ being kept fixed, we omit it in the following). 


$$
\begin{array}{lr}
a_{n}(\lambda)=\frac{1}{2 \pi} \int_{0}^{2 \pi} \Psi(\phi, \lambda) e^{-i n \phi} d \phi=\frac{1}{\pi} \int_{0}^{\pi} \Psi(\phi, \lambda) \cos n \phi d \phi, \\
a_{n}(\lambda) \equiv a_{-n}(\lambda) & (n=0,1,2, \cdots)\left({ }^{9}\right) .
\end{array}
$$

$a_{n}(\lambda)$ possesses an analytic continuation, which is regular except possibly on the interval $0, \infty$, since it is $\left(\lambda_{\sigma, \tau}(m)\right)^{-1 / 2}$ times $\left(R(\lambda) f, e_{m}\right)_{\sigma, \tau}$ (for the definition of $e_{m}$, cf. 2.a). Next we shall find an analytic expression for $a_{n}(\lambda)$, valid for each $\lambda$ such that $\operatorname{Re} \lambda<1 / 4$ or $\operatorname{Im} \lambda>0$; we denote this domain by $D_{0}$ in the following. First we write $\Psi(\phi, \lambda)$ in the form

$$
(1-\cos \phi)^{-(\sigma+\tau) / 2-1 / 4+\mu / 2} \Psi_{1}(\phi, \lambda) .
$$

Suppose that $f(\phi)$ vanishes if $0<\phi \leqq \delta$. Then for such a $\phi$, taking in view that for $0<\phi \leqq \pi$

$$
g_{1}(\phi, \lambda)=(1-\cos \phi)^{-1 / 4+\mu / 2} F\left(a, b, c, \sin ^{2} \phi / 2\right),
$$

we have

$$
\Psi_{1}(\phi, \lambda) \equiv F\left(a, b, c, \sin ^{2} \phi / 2\right) / D(\lambda) \int_{0}^{2 \pi}(a(\psi))^{-1} g_{1}(\psi, \lambda) f(\psi) d \psi
$$

from which it follows immediately that $\Psi_{1}^{\prime}(0, \lambda) \equiv 0$. Putting $a(\lambda)=\Psi_{1}(0, \lambda)$ and $\Phi(\phi, \lambda)=\Psi_{1}(\phi, \lambda)-a(\lambda)$, we have

$$
a_{n}(\lambda)=a_{n}^{(1)}(\lambda)+a_{n}^{(2)}(\lambda)
$$

where

$$
a_{n}^{(1)}(\lambda)=a(\lambda) / \pi \int_{0}^{\pi}(1-\cos \phi)^{-(\sigma+r) / 2-1 / 4+\mu / 2} \cos n \phi d \phi
$$

and

$$
a_{n}^{(2)}(\lambda)=1 / \pi \int_{0}^{\pi}(1-\cos \phi)^{-(\sigma+\tau) / 2-1 / 4+\mu / 2} \Phi(\phi, 2) \cos n \phi d \phi(n=0,1,2, \cdots) .
$$

We are going to discuss these two expressions separately.

For $\operatorname{Re} s>0$ we have the formula

$$
\frac{1}{\pi} \int_{0}^{\pi}(1-\cos \phi)^{--1 / 2} \cos n \phi d \phi=\frac{2^{s-1 / 2} B(s, 1 / 2)}{\pi} \lambda_{m}(s)
$$

where, as before,

$$
B(x, y)=\frac{\Gamma(x) \Gamma(y)}{\Gamma(x+y)}
$$

(9) We suppose (cf. footnote 8 ) that in these formulas $\lambda$ is restricted to an appropriate domain, such that $\Psi_{j}(\phi \lambda)$ is integrable. 
and

$$
\lambda_{0}(s) \equiv 1, \quad \lambda_{m}(s)=\prod_{j=1}^{m}\left(\frac{j-1 / 2-s}{j-1 / 2+s}\right) \quad(m=1,2, \cdots) .
$$

Hence, supposing $\lambda$ chosen in such a way that

$$
\operatorname{Re}\left(-\frac{1}{2}(\sigma+\tau)+\frac{1}{4}+\frac{1}{2} \mu\right)>0
$$

we have

$$
a_{m}^{(1)}(\lambda)=\frac{a(\lambda) 2^{s-1 / 2} B(s, 1 / 2)}{\pi} \lambda_{m}(s)
$$

with $s=-(\sigma+\tau) / 2+1 / 4+\mu / 2$. But the factor of $a(\lambda)$ in this expression for $a_{m}^{(1)}(\lambda)$ is an analytic function of $\lambda$ in $D_{0}$, except possibly for one pole, when $s=0$, since always $\operatorname{Re} s>-1 / 2$. On the other hand we have

$$
a(\lambda)=1 / D(\lambda) \int_{0}^{2 \pi}(a(\psi))^{-1} g_{2}(\psi, \lambda) f(\psi) d \psi
$$

To make it easier to prove that expressions like this are analytic, we recall the following well-known facts. We denote by $u_{1}(\phi, \lambda)$ and $u_{2}(\phi, \lambda)$ those solutions of the equation $L^{0} u+\lambda u / 2=0$, for which

$$
u_{1}(\phi, \lambda) \equiv 1, \quad u_{1}^{\prime}(\phi, \lambda) \equiv 0
$$

and

$$
u_{2}(\phi, \lambda) \equiv 0, \quad u_{2}^{\prime}(\phi, \lambda) \equiv 1 .
$$

Then $u_{i}(\phi, \lambda)(i=1,2)$ are continuous jointly in $\phi$ and $\lambda$, if the former varies over a closed subset of the interval $(0,2 \pi)$. Moreover, $u_{i}(\phi, \lambda)$ $(i=1,2)$ is an entire function of $\lambda$ for any fixed $0<\phi<2 \pi$. In our case we have

$$
g_{1}(\phi, \lambda) \equiv g_{1}(\pi, \lambda) u_{1}(\phi, \lambda)+g_{1}^{\prime}(\pi, \lambda) u_{2}(\phi, \lambda)
$$

and

$$
g_{2}(\phi, \lambda) \equiv g_{1}(\pi, \lambda) u_{1}(\phi, \lambda)-g_{1}^{\prime}(\pi, \lambda) u_{2}(\phi, \lambda) .
$$

Since $D(\lambda)=-4 g_{1}(\pi, \lambda) g_{1}^{\prime}(\pi, \lambda)$ (cf. B3), we have

$$
\begin{aligned}
a(\lambda)= & -1 / 4 g_{1}^{\prime}(\pi, \lambda) \int_{0}^{2 \pi}(a(\psi))^{-1} u_{1}(\psi, \lambda) f(\psi) d \psi \\
& +1 / 4 g_{1}(\pi, \lambda) \int_{0}^{2 \pi}(a(\psi))^{-1} u_{2}(\psi, \lambda) f(\psi) d \psi .
\end{aligned}
$$


After this, in order to prove that $a(\lambda)$ is analytic in $D_{0}$ it suffices to remark that in this case both $\operatorname{Re}(3 / 4 \pm(\sigma-\tau) / 2+\mu / 2)$ and $\operatorname{Re}(1 / 4 \pm(\sigma-\tau) / 2+\mu / 2)$ are positive, hence (cf. the expressions for $g_{1}(\pi, \lambda)$ and $g_{1}^{\prime}(\pi, \lambda)$ in $\left.(3)\right) 1 / g_{1}(\pi, \lambda)$ and $1 / g_{1}^{\prime}(\pi, \lambda)$ are regular.

For later use observe that $a(\lambda)$ remains bounded if $0<\lambda<1 / 4, \lambda \rightarrow 1 / 4$.

Thus we have shown that $a_{m}^{(1)}(\lambda)$ is analytic in $D_{0}$, except possibly for one pole in the interval $(0,1 / 4)$.

Next we are going to discuss the expression

$a_{m}^{(2)}(\lambda)=\frac{1}{\pi} \int_{0}^{\pi}(1-\cos \phi)^{-(\sigma+\tau) / 2-1 / 4+\mu / 2} \Phi(\phi, \lambda) \cos m \phi d \phi \quad(m=0,1, \cdots)$.

First of all, we observe that this expression exists for any $\lambda \in D_{0}$. For if $0<\phi<\delta$ we have

$$
\Phi(\phi, \lambda)=\left(F\left(a, b, c, \sin ^{2} \phi / 2\right)-1\right) a(\lambda)=O\left(\phi^{2}\right)
$$

and $\operatorname{Re}(-(\sigma+\tau) / 2-1 / 4+\mu / 2)>-3 / 4$. Let us suppose that we have chosen $\epsilon>0$ in such a way that $0<\epsilon<\delta$, and a bounded domain $D$ with a closure $\bar{D} \subset D_{0}$. We put

$$
F_{1}(\lambda, \epsilon, m)=\frac{1}{\pi} \int_{0}^{e}(1-\cos \phi)^{-(\sigma+\tau) / 2-1 / 4+\mu / 2} \Phi(\phi, \lambda) \cos m \phi d \phi
$$

and

$$
F_{2}(\lambda, \epsilon, m)=\frac{1}{\pi} \int_{\epsilon}^{\pi}(1-\cos \phi)^{-(\sigma+\tau) / 2-1 / 4+\mu / 2} \Phi(\phi, \lambda) \cos m \phi d \phi .
$$

We recall that $\Phi(\phi, \lambda)=\Psi_{1}(\phi, \lambda)-a(\lambda)$, where $a(\lambda)$ is analytic in $D_{0}$, and that

$$
\begin{aligned}
(1-\cos \phi)^{-1 / 4+\mu / 2} \psi_{1}(\phi, \lambda)= & 1 / D(\lambda)\left(g_{2}(\phi, \lambda) \int_{0}^{\phi}(a(\psi))^{-1} f(\psi) g_{2}(\psi, \lambda) d \psi\right. \\
& \left.+g_{1}(\phi, \lambda) \int_{\phi}^{2 \pi}(a(\psi))^{-1} f(\psi) g_{2}(\psi, \lambda) d \psi\right) .
\end{aligned}
$$

Making use of the expression of $g_{i}(\phi, \lambda)$ by aid of the functions $u_{i}(\phi, \lambda)(i=1,2)$ described above, we may conclude that the integrand in $F_{2}(\lambda, \epsilon, m)$ is of the following type. It is a sum of expressions of the form

$$
v_{1}(\phi, \lambda) \int_{0}^{\phi} f_{1}(\psi) w_{1}(\psi, \lambda) d \psi
$$

and

$$
v_{2}(\phi, \lambda) \int_{\phi}^{2 \pi} f_{2}(\psi) w_{2}(\psi, \lambda) d \psi \quad(\phi \geqq \epsilon)
$$


where $f_{i}(\phi)$ are continuous and vanish if $0 \leqq \phi \leqq \delta ; v_{i}(\phi, \lambda)$ and $w_{i}(\phi, \lambda)(i=1,2)$ are continuous if the pair $(\phi, \lambda)$ varies over the product of the interval $[\epsilon, \pi]$ with $\bar{D}$; finally, for each fixed $\epsilon \leqq \phi \leqq \pi, v_{i}(\phi, \lambda)$ and $w_{i}(\phi, \lambda)(i=1,2)$ are regular functions of $\lambda$ in $D_{0}$. But from this it easily follows that for each $0<\epsilon$ and $m F_{2}(\lambda, \epsilon, m)$ is regular too in every point of $D_{0}$.

Next we turn to $F_{1}(\lambda, \epsilon, m)$. We write

$$
F(a, b, c, x)-1=F^{\prime}\left(a, b, c, x^{\prime}\right) x \quad\left(0<x^{\prime}<x\right) .
$$

Suppose we have already proved that $\left|F^{\prime}(a, b, c, x)\right| \leqq M$, if $\lambda$ varies in $D$ and $0<x<\gamma, \gamma$ fixed, with an $M$ depending on $D$ only. Then we have the following estimate for $F_{1}(\lambda, \epsilon, m)$ :

$$
\left|F_{1}(\lambda, \epsilon, m)\right| \leqq \frac{M}{2 \pi} \int_{0}^{\epsilon}(1-\cos \phi)^{1 / 4+(\operatorname{Re} \mu) / 2} d \phi
$$

which tends to 0 with $\epsilon$, uniformly in $\lambda \in D$. Since $a_{m}^{(2)}(\lambda)=F_{1}(\lambda, \epsilon, m)$ $+F_{2}(\lambda, \epsilon, m)$, this proves that $a_{m}^{(2)}(\lambda)$, and hence $a_{m}(\lambda)=a_{m}^{(1)}(\lambda)+a_{m}^{(2)}(\lambda)$ is regular in $D_{0}$ for each $m=0,1,2, \cdots$.

In order to prove the statement concerning $F^{\prime}(a, b, c, x)$ used above, we recall that for $|z|<1$ we have [2, p. 249]:

$$
F(a, b, c, z)=\frac{\Gamma(c)}{\Gamma(b) \Gamma(c-b)} \int_{0}^{1} t^{b-1}(1-t)^{c-b-1}(1-z t)^{-a} d t
$$

provided that $\operatorname{Re} c>\operatorname{Re} b>0$. This condition is fulfilled in our case, since $\operatorname{Re}(c-b)=\operatorname{Re}(3 / 4+(\sigma-\tau) / 2+\mu / 2) \geqq 1 / 2$ if $\lambda \in D_{0}$. From this formula we get

$$
F^{\prime}(a, b, c, z)=\frac{a \Gamma(c)}{\Gamma(b) \Gamma(c-b)} \int_{0}^{1} t^{b}(1-t)^{c-b-1}(1-z t)^{-a-1} d t .
$$

The factor of the integral in this expression is a continuous function of $\lambda$, if it varies over $D$; denote the upper bound of its absolute value by $M_{1}$. Putting moreover $\alpha$ and $\beta$ for sup $\operatorname{Re} a$ and $\inf (1$, inf $\operatorname{Re}(c-b))(\lambda \in D)$ resp., we have

$$
\left|F^{\prime}(a, b, c, x)\right| \leqq M_{1} \int_{0}^{1}(1-t)^{\beta-1}(1-\gamma t)^{-\alpha-1} d t=M
$$

$(0 \leqq x \leqq \gamma ; \lambda \in D)$, where $M$ depends on $D$ only, which proves our statement.

For later use observe the following two facts, which are easy consequences of the above discussion. First, given any compact set $C$ inside $D_{0}$, we have $\left|a_{m}^{(2)}(\lambda)\right| \leqq K$, where $K$ depends on $C$ but not on $m$. Secondly, for each $m, a_{m}^{(2)}(\lambda)$ remains bounded if $\lambda \rightarrow 1 / 4$ from the left along the real line.

Summing up, we have $a_{m}(\lambda) \equiv\left(R(\lambda) f, e_{m}\right)_{o \tau}\left(\lambda_{\sigma \tau}(m)\right)^{-1 / 2}$ for $\operatorname{Im} \lambda>0$, and

$$
a_{m}(\lambda) \equiv \frac{a(\lambda) 2^{s-1 / 2} B(s, 1 / 2)}{\pi} \lambda_{m}(s)+a_{m}^{(2)}(\lambda) \quad(m=1,2, \cdots)
$$


where $s=-(\sigma+\tau) / 2+1 / 4+\mu / 2$, and $a_{m}(\lambda)$ is regular in $D_{0}$, except possibly for $s=0$.

To obtain an expression for $h_{f}(\lambda)$, which gives the analytic continuation of $(R(\lambda) f, f)_{\sigma, \tau}$ over $D_{0}$, observe that we have

$$
n^{2 z} \prod_{j=1}^{n+1}\left(\frac{j-1 / 2-z}{j-1 / 2+z}\right)=\frac{\Gamma(1 / 2+z)}{\Gamma(1 / 2-z)}\left(1+\epsilon_{n}(z)\right),
$$

where $\epsilon_{n}(z) \rightarrow 0$ if $n \rightarrow+\infty$, uniformly on any compact subset of the complex plane, not containing the points $z_{k}=-(k+1 / 2)(k=0,1,2, \cdots)$. Since $\operatorname{Re} s=-\operatorname{Re}(\sigma+\tau) / 2+1 / 4+(\operatorname{Re} \mu) / 2>-1 / 4$, we have for any compact subset $C \subset D_{0}\left|\lambda_{n}(s)\right|<K(C) n^{1 / 2}(n=1,2, \cdots)$, where $K(C)$ does not depend on $n$. Suppose now that we have

$$
f(\phi)=\sum_{-\infty}^{\infty} a_{n} e^{i n \phi}
$$

We recall that by virtue of our assumption, according to which $f(\phi)$ is indefinitely differentiable, if $n \rightarrow+\infty a_{n} \rightarrow 0$ faster than any negative power of $n$, and that $\lambda_{\sigma, \tau}(m) \leqq 1$. Therefore the following two series

$$
f_{1}(\lambda)=\frac{a(\lambda) 2^{s-1 / 2}}{\pi} \sum_{-\infty}^{\infty} \lambda_{m}(s) \bar{a}_{m} \lambda_{\sigma, \tau}(m)
$$

and

$$
f_{2}(\lambda)=\sum_{-\infty}^{\infty} a_{m}^{(2)}(\lambda) \bar{a}_{m} \lambda_{\sigma, \tau}(m)
$$

represent regular functions in $D_{0}$. Putting

$$
h_{f}(\lambda)=B(s, 1 / 2) f_{1}(\lambda)+f_{2}(\lambda),
$$

we have $h_{f}(\lambda)=(R(\lambda) f, f)_{\sigma, \tau}$ for $\operatorname{Im} \lambda>0$; therefore it is this function which gives the desired analytic continuation over the whole of $D_{0}$. It is regular everywhere, except possibly for that $\lambda_{0}, 0<\lambda_{0}<1 / 4$, for which $s=0$.

Before continuing the discussion of the spectrum of $Q_{0}^{+}$, we recall the following well-known theorem (cf. $[13,3.5$, p. 47]):

Let

$$
F(\lambda)=\int_{-\infty}^{\infty} \frac{d \sigma(t)}{t-\lambda}
$$

where $\sigma(t)$ is of a bounded variation over the real line. Then $F(\lambda)$ is an analytic function of $\lambda$ on the upper and lower half-plane, and for $t_{1}<t_{2}$ we have

$$
\frac{1}{\pi} \lim _{\delta \rightarrow 0} \int_{t_{1}}^{t_{2}} \operatorname{Im} F(t+i \delta) d t=\sigma\left(t_{2}-0\right)-\sigma\left(t_{1}+0\right) .
$$


Furthermore, let us recall that for $f \in C$ we have $(f, f)_{\sigma, \tau} \leqq(1 / 2 \pi) \int_{0}^{2 \pi}|f(\phi)|^{2} d \phi$, which implies that $C$ is dense in $H_{\sigma, \tau}$ (and hence in $H_{\sigma, \tau}^{+}$and $H_{\sigma, \tau}^{-}$) along with $L^{2}\left(T^{1}\right)$. Next we put $Q_{0}^{+}=\int_{-\infty}^{\infty} t d E_{t}$, and consider the following two cases.

$\left(\beta_{1}\right) \operatorname{Re}(\sigma+\tau) \leqq 1 / 2$. In this case $\operatorname{Re} s>0$ if $0 \leqq \lambda<1 / 4$, hence $h_{f}(\lambda)$ is regular everywhere in $D_{0}$. Let us choose $u$ such that $0<u<1 / 4$. Since

$$
h_{f}(\lambda)=\int_{-\infty}^{\infty} \frac{d\left\|E_{t} f\right\|_{\sigma \tau}^{2}}{t-\lambda}
$$

we have by the theorem quoted above

$$
\left\|E_{u-0} f\right\|_{\sigma, \tau}^{2}=\frac{1}{\pi} \lim _{\delta \rightarrow 0} \int_{-u}^{u} \operatorname{Im} h_{f}(t+i \delta) d t=0,
$$

since from $\operatorname{Im} h_{f}(\lambda) \equiv \operatorname{Im}(R(\lambda) f, f)_{\sigma, \tau} \equiv 0$ for $\lambda<0$, it follows the same for $0 \leqq \lambda<1 / 4$, and by virtue of $Q_{0}^{+} \geqq 0, E_{-u}=0$ for $u>0$. Since $C$ is dense in $H_{\sigma, \tau}^{+}$ and $u$ was arbitrary $0<u<1 / 4$, we may conclude that if $\operatorname{Re}(\sigma+\tau) \leqq 1 / 2$, then the spectrum of $Q_{0}^{+}$is empty in the interval $0 \leqq t<1 / 4$.

$\left(\beta_{2}\right) \sigma+\tau>1 / 2$. In this case the equation $0=s=-(\sigma+\tau) / 2+1 / 4+\mu / 2$ has the solution $0<1 / 4-(\sigma+\tau-1 / 2)^{2}<1 / 4$, at which point $B(s, 1 / 2)$ has a simple pole. Since for $\lambda \neq \lambda_{0} h_{f}(\lambda)$ is regular, we may conclude as above that $E_{t}$ is constant for $0<t<1 / 4$, except possibly for a jump at $t=\lambda_{0}$. To see that this jump does exist, consider the relation

$$
\left(R(\lambda) f, e_{0}\right)=\frac{a(\lambda) 2^{s-1 / 2} B(s, 1 / 2)}{\pi}+a_{0}^{(2)}(\lambda) \quad\left(\lambda \in D_{0}\right)
$$

deduced above. This expression can be regular at $\lambda_{0}$ for a fixed $f \in C$, only if we have

$$
0=a\left(\lambda_{0}\right)=1 / D\left(\lambda_{0}\right) \int_{0}^{2 \pi}(a(\psi))^{-1} g_{2}\left(\psi, \lambda_{0}\right) f(\psi) d \psi .
$$

We know that $D(\lambda)$ is regular in $D_{0}$ and therefore the factor of the integral in the above expression cannot be 0 . Hence from the validity of the above relation for any $f \in C, f^{T}=f$ it follows that $g_{2}\left(\pi, \lambda_{0}\right)=g_{1}\left(\pi, \lambda_{0}\right)=0$, which is impossible (cf. the expression for $g_{1}(\pi, \lambda)$ in B3).

Observe that by $(\alpha)$ the eigenspace belonging to $\lambda_{0}$ is one-dimensional. Summing up: If $\sigma+\tau>1 / 2$, then the spectrum of $Q_{0}^{+}$in the interval $0 \leqq t<1 / 4$ consists of a simple eigenvalue $0<\lambda_{0}<1 / 4$ determined by $\lambda_{0}=1 / 4-(\sigma+\tau-1 / 2)^{2}$.

$(\gamma)$ Our next objective will be the discussion of the spectrum of $Q_{0}^{+}$in the interval $[1 / 4,+\infty]$. First we prove that every point of this interval belongs to the spectrum of $Q_{0}^{+}$. The idea of the proof is as follows. We show that for any $f \in C \cap H_{\sigma, \tau}^{+}$the function defined for $\operatorname{Im} \lambda>0$ by $(R(\lambda) f, f)_{\sigma, \tau}$ possesses an analytic continuation $h_{f}(\lambda)$, which is regular at every point of the interval $[1 / 4,+\infty]$. Assuming this assertion proved, we show that the above state- 
ment concerning the spectrum of $Q_{0}^{+}$is a simple consequence of it. As a matter of fact, we can assume $1 / 4<t_{1}<t_{2}$ to be chosen in such a way that $E_{t_{2}-0}-E_{t_{1}+0} \neq 0$; for otherwise $E_{t}$ would be constant, except for at most two jumps $0<\lambda_{0}<1 / 4$ and $1 / 4$, which is impossible by $(\alpha)$. We have

$$
\left\|\left(E_{t_{2}-0}-E_{t_{1}+0}\right) f\right\|^{2}=\frac{1}{\pi} \lim _{\delta \rightarrow 0} \int_{t_{1}}^{t_{2}} \operatorname{Im} h_{f}(t+i \delta) d t=\frac{1}{\pi} \int_{t_{1}}^{t_{2}} v_{f}(t) d t,
$$

where $v_{f}(t) \equiv \operatorname{Im} h_{f}(t) \geqq 0$. By a former remark $C$ is dense in $H_{\sigma, r}^{+}$, hence for an appropriate choice of $f$, the left-hand side of the above equation differs from 0 . Therefore in this case $v_{f}(t)$ does not vanish identically when $1 / 4<t<$ $+\infty$. Moreover, since by our assumption $h_{f}(\lambda)$ is regular at every point of this interval, $v_{f}(t)$ is positive except possibly for a finite number of points in every closed interval $\left[t_{1}, t_{2}\right]$ with $1 / 4<t_{1}<t_{2}$. This evidently proves our statement concerning the spectrum of $Q_{0}^{+}$.

Actually this reasoning shows that the spectrum of $Q_{0}^{+}$is equivalent to the Lebesgue measure in the interval $[1 / 4,+\infty]$. For this we recall that given a self-adjoint operator $A=\int_{-\infty}^{\infty} t d E_{t}$ on a separable Hilbert space $H$, its spectrum is called equivalent to the Lebesgue measure if a Borel set $E$ is of a (Lebesgue) measure 0 , if and only if it is of a measure 0 with respect to all measures, corresponding to the nondecreasing functions $\left\|E_{t} f\right\|^{2}(f \in H,-\infty<t<+\infty)$. It is not hard to show that the spectrum of $A$ is equivalent to the Lebesgue measure, even if in the above definition we restrict ourselves to a family of functions $\left\{\left\|E_{\imath} f\right\|^{2}\right\}$, where $f$ runs over a set $H^{\prime} \subset H$, which is dense in $H$.

Since $C$ is dense in $H_{\sigma, \tau}^{+}$, our assertion concerning the nature of the spectrum of $Q_{0}^{+}$becomes clear.

Now we turn to the proof of the fact that $(R(\lambda) f, f)_{\sigma, \tau}\left(\operatorname{Im} \lambda>0, f \in C \cap H^{+}\right)$ possesses an analytic continuation $h_{f}(\lambda)$, which is regular at every point $1 / 4<\lambda<+\infty$. We recall that according to our choice of $\mu$ as a branch of $(1 / 4-\lambda)^{1 / 2}, \operatorname{Re} \mu>0$ if $\operatorname{Im} \lambda>0$ and $\leqq 0$ if $\lambda=x+i y, y \leqq 0, x>1 / 4$. In other words $\operatorname{Re} \mu$ changes its sign from the positive to the negative if $\lambda$ crosses the interval $[1 / 4,+\infty]$ in the direction of the half-plane $\operatorname{Im} \lambda<0$. We choose a point $\lambda_{0}, 1 / 4<\lambda_{0}<+\infty$, which we keep fixed in the following. Next we resume the reasonings of $(\beta)$ step by step, and show that the $h_{f}(\lambda)$, regular around $\lambda_{0}$ can be constructed in a similar fashion as it was done in $(\beta)$. We use the notations of $(\beta)$ without further explanation, and whenever possible, avoid unnecessary repetitions.

We again consider the expressions

$$
\begin{aligned}
\left(\lambda_{\sigma, \tau}(m)\right)^{-1 / 2}\left(R(\lambda) f, e_{m}\right) & \equiv a_{m}(\lambda) \equiv a_{m}^{(1)}(\lambda)+a_{m}^{(2)}(\lambda), \quad(m=0,1, \cdots) \\
a_{m}(\lambda) & \equiv a_{-m}(\lambda) .
\end{aligned}
$$

We know that the functions $a_{m}^{(i)}(\lambda)(i=1,2)$ are regular for $\operatorname{Im} \lambda>0$. In particular, we have 


$$
a_{m}^{(1)}(\lambda)=\frac{a(\lambda) 2^{s-1 / 2} B(s, 1 / 2)}{\pi} \lambda_{m}(s) .
$$

Since $\mu$ is purely imaginary for $\lambda_{0}$, we have in this case $\operatorname{Re} s>0$, and $\operatorname{Re}(1 / 2 \pm(\sigma-\tau) / 2+\mu / 2), \operatorname{Re}(3 / 4 \pm(\sigma-\tau) / 2+\mu / 2)>0$. From this it is clear that if $\lambda$ varies in a sufficiently small neighborhood of $\lambda_{0}, 1 / g_{1}(\pi, \lambda)$ and $1 / g_{1}^{\prime}(\pi, \lambda)$, and hence $a_{m}^{(1)}(\lambda)$ is a regular function of $\lambda$. Moreover, for $\operatorname{Im} \lambda>0$ the latter coincides with its definition given in $(\beta)$.

In the same way we can show that $a_{m}^{(2)}(\lambda)$ depends regularly on $\lambda$, provided that $\lambda$ is close to $\lambda_{0}$ in such a way that $\operatorname{Re} \mu>-\gamma$, where $\gamma>0$ is a suitable constant. We have $\left|a_{m}^{(2)}(\lambda)\right|<K$ everywhere in this neighborhood, with a $K$ not depending on $m$ and $\lambda$, and for $\operatorname{Im} \lambda>0, a_{m}^{(2)}(\lambda)$ again is identical with its previous definition.

Finally, under the same restrictions on $\lambda$ we have an estimate $\left|\lambda_{n}(s)\right|$ $<K n^{k}$, where $K, k>0$ do not depend on $n$.

Summing up, if

$$
f(\phi)=\sum_{-\infty}^{\infty} a_{n} e^{i n \phi}
$$

then the series

$$
\sum_{-\infty}^{\infty} a_{m}(\lambda) \bar{a}_{m} \lambda_{\sigma, \tau}(m)
$$

is a uniformly convergent series of functions, analytic if $\left|\lambda-\lambda_{0}\right|<\delta$, where $\delta$ is sufficiently small. Denoting its sum by $h_{f}(\lambda)$, it is clear that for $\operatorname{Im} \lambda>0$ it is identical with $(R(\lambda) f, f)_{\sigma, \tau}$, and thus it gives an analytic continuation of it, if $\operatorname{Im} \lambda \leqq 0$.

Since $\lambda_{0}$ was arbitrary in the interval $(1 / 4,+\infty)$, the existence of the analytic continuation of $(R(\lambda) f, f)_{\sigma, \tau}$ with the required properties has been established.

In order to complete the discussion of the spectrum of $Q_{0}^{+}$, we have to show that $E_{t}$ is continuous at the point $1 / 4$. This question has still been left open.

Assuming the opposite, we put $P_{0}=E_{1 / 4+0}-E_{1 / 2-0}$, and we can suppose that $f \in C \cap H^{+}$and $m$ have been chosen in such a way that $\left(P_{0} f, e_{m}\right)_{\sigma, r} \neq 0$. By virtue of $(\alpha)$ and $(\beta)$ we have for $0<\lambda<1 / 4, \lambda \neq \lambda_{0}$

$$
\left(R(\lambda) f, e_{m}\right)_{\sigma, \tau}=\frac{a_{1}}{\lambda_{0}-\lambda}+\frac{a_{2}}{1 / 4-\lambda}+\int_{1 / 4}^{\infty} \frac{v(t)}{t-\lambda} d t
$$

where $a_{2}=\left(P_{0} f, e_{m}\right)_{\sigma, \tau}$, and $v(t)$ is a function, integrable over $[1 / 4,+\infty]$, depending on $f$ and $m$. Hence in order to arrive at a contradiction, it suffices to prove that 


$$
\lim _{\lambda \rightarrow 1 / 4-0}(\lambda-1 / 4)\left(R(\lambda) f, e_{m}\right)_{\sigma, \tau}=0 .
$$

But (cf. $(\beta)$ ) this amounts to showing that

$$
\lim _{\lambda \rightarrow 1 / 4-0}(1 / 4-\lambda)\left[\frac{a(\lambda) 2^{s-1 / 2} B(s, 1 / 2)}{\pi}+a_{m}^{(2)}(\lambda)\right]=0 .
$$

By remarks made during the discussion of $a(\lambda)$ and $a_{m}^{(2)}(\lambda)$ we know that they remain bounded when $\lambda$ approaches $1 / 4$ from the left. Therefore it suffices to prove that $\lim _{\lambda \rightarrow 1 / 4-0}(1 / 4-\lambda) B(s, 1 / 2)=0$. Since $s=-(\sigma+\tau) / 2+1 / 4+$ $\mu / 2$, this is clear if $(\sigma+\tau) / 2 \neq 1 / 4$; but even in the remaining case we have $(1 / 4-\lambda) B(s, 1 / 2)=O\left((1 / 4-\lambda)^{1 / 2}\right)$, which completes the proof.

Putting together the results of B4.a $(\alpha)-(\gamma)$ we have obtained the following: The part $Q_{0}^{+}$of $Q$ in the subspace $H_{\sigma, \tau}^{+}$of $H_{\sigma, \tau}$ has a simple spectrum, which is equivalent to the Lebesgue measure over the interval $[1 / 4,+\infty]$. The number $1 / 4$ cannot be an eigenvalue of $Q_{0}^{+}$. The spectrum of $Q_{0}^{+}$is empty in $(-\infty, 1 / 4)$, except when $\sigma+\tau>1 / 2$, in which case $Q_{0}^{+}$has an eigenvalue $\lambda_{0}=1 / 4-$ $(\sigma+\tau-1 / 2)^{2}$.

(b) Case of $Q_{0}^{-}$. In order to complete the discussion of the spectrum of $Q_{0}$ in $H_{\sigma, \tau}$, we now consider its part $Q_{0}^{-}$in $H_{\sigma, \tau}^{-}$. Since much of what follows is closely parallel to the corresponding steps in (a), we can sometimes be quite short.

$(\alpha)$ In order to show that the spectrum of $Q_{0}^{-}$is simple, it suffices to prove that the linear span of the system $\left(Q_{0}\right)^{n} f_{0}(n=1,2, \cdots)$ where $f_{0}(\phi) \equiv \sin m \phi$ $\in H_{\sigma, \tau}^{-}$, contains the system $\{\sin m \phi\}$. For this it is enough to show that $Q \sin \nu \phi$ is an odd trigonometric polynomial of degree $\nu+1$. Assuming the statement true for $1 \leqq \nu \leqq m-1(m>1)$, an elementary computation shows that $Q \sin m \phi$ is odd and at most of a degree $m+1$; moreover the coefficient of $\sin (m+1) \phi$ is $-(m+1 / 2+\sigma)(m+1 / 2+\tau) / 4 \neq 0$, which completes the proof.

( $\beta$ ) Since $Q_{0}^{-} \geqq 0$, when discussing the part of its spectrum in $(-\infty, 1 / 4)$, we may restrict ourselves to the interval $0 \leqq \lambda<1 / 4$. Next we show that for $f \in H^{-} \cap C$, the function defined for $\operatorname{Im} \lambda \gtrless 0$ and $\lambda<0$ by $(R(\lambda) f, f)_{\sigma, \tau}$ possesses a continuation $h_{f}(\lambda)$, which is regular for $0 \leqq \lambda<1 / 4$. Since $h_{f}(\lambda)$ is real for $\lambda<0$, so is it in $[0,1 / 4)$. Proceeding as in a. $\beta$, this result yields

$$
\left.\left\|E_{u-0} f\right\|_{\sigma, \tau}^{2} \lim _{\delta \rightarrow 0} \frac{1}{\pi} \int_{-u}^{u} \operatorname{Im}(R(t+i \delta) f, f)_{\sigma, \tau}\right) d t=0
$$

for any $f \in C \cap H^{-}$and $0<u<1 / 4$, provided that $Q_{0}^{-}=\int_{-\infty}^{\infty} t d E_{t}$, which implies $E_{t}=0$ for $t<1 / 4$. In other words, the spectrum of $Q_{0}^{-}$is empty in the interval $(-\infty, 1 / 4)$.

In order to obtain the required continuation, we are again going to study the Fourier coefficients of $\Psi(\phi, \lambda)$, corresponding to a fixed $f \in C, f^{T}=-f$. We 
know that for $\operatorname{Re} \mu$ sufficiently large $\Psi(\phi, \lambda)$ is square-integrable, and $R(\lambda) f=$ $\Psi(\phi, \lambda)$ (cf. B3). Furthermore in our case we have $\Psi^{T} \equiv-\Psi$. Therefore, writing as in a. $\beta$

$$
\Psi(\phi, \lambda) \equiv(1-\cos \phi)^{-(\sigma-\tau) / 2-1 / 4+\mu / 2} \Psi_{1}(\phi, \lambda) \quad(0<\phi<2 \pi)
$$

we get

$$
\begin{array}{rlr}
a_{m}(\lambda) & =\frac{1}{2 \pi} \int_{0}^{2 \pi} \Psi(\phi, \lambda) e^{-i m \phi} d \phi & (m=1,2, \\
& =\frac{1}{\pi i} \int_{0}^{\pi}(1-\cos \phi)^{-(\sigma+\tau) / 2-1 / 4+\mu / 2} \Psi_{1}(\phi, \lambda) \sin m \phi d \phi
\end{array}
$$

and $a_{-m}(\lambda) \equiv-a_{m}(\lambda)$.

Observe that the expression on the right-hand side exists for any $\lambda$ in $D_{0}$, where the latter again denotes the union of the half-planes $\operatorname{Im} \lambda>0$ and $\operatorname{Re} \lambda<1 / 4$. To see this, it suffices to remark that $\Psi_{1}(\phi, \lambda)$ is continuous at $\phi=0$, and the function $(1-\cos \phi)^{-(\sigma+\tau) / 2-1 / 4+\mu / 2} \sin m \phi$ is integrable over $[0, \pi]$. Moreover, for $\operatorname{Im} \lambda>0$ we have $a_{m}(\lambda)=\left(R(\lambda) f, e_{m}\right)_{\sigma, \tau}\left(\lambda_{\sigma \tau}(m)\right)^{-1 / 2}$. Next we show that $a_{m}(\lambda)$ depends regularly on $\lambda$ in the neighborhood of any $0 \leqq \lambda_{0}<1 / 4$. The proof is almost identical with that proving the analyticity of $a_{m}^{(2)}(\lambda)$ in the same interval given in a. $\beta$.

Suppose that $f(\phi)$ vanishes for $0 \leqq \phi \leqq \delta$; choosing $0<\epsilon<\delta$ we put

$$
\begin{aligned}
& F_{1}(\lambda, \epsilon, m)=\frac{1}{\pi i} \int_{0}^{e}(1-\cos \phi)^{-(\sigma+\tau) / 2-1 / 4+\mu / 2} \Psi_{1}(\phi, \lambda) \sin m \phi d \phi, \\
& F_{2}(\lambda, \epsilon, m)=\frac{1}{\pi i} \int_{0}^{\pi}(1-\cos \phi)^{-(\sigma+\tau) / 2-1 / 4+\mu / 2} \Psi_{1}(\phi, \lambda) \sin m \phi d \phi .
\end{aligned}
$$

The arguments for the analyticity of $F_{2}(\lambda, \epsilon, m)$ around any $\lambda_{0} \in D_{0}$ are the same as those for the analogous expression in a. $\beta$ and will not be repeated. To complete the proof of our statement, it suffices to show that for any compact set $C \subset D_{0}\left|\Psi_{1}(\phi, \lambda)\right| \leqq M$, if $0 \leqq \phi \leqq \epsilon$ and $\lambda \in C$, where $M$ depends on $C$ only. Since in this case

$$
\Psi_{1}(\phi, \lambda) \equiv F\left(a, b, c, \sin ^{2} \phi / 2\right) / D(\lambda) \int_{0}^{2 \pi}(a(\psi))^{-1} f(\psi) g_{2}(\psi, \lambda) d \psi
$$

and the factor of $F$ is an analytic function of $\lambda$ in $D_{0}$, it is enough to show that

$$
|F(a, b, c, x)| \leqq M,
$$

if $0 \leqq x \leqq \gamma, \gamma$ fixed, and $\lambda \in C$, with an $M$ depending on $C$ only. But this is easily achieved by aid of the integral representation

$$
F(a, b, c, z)=\frac{\Gamma(c)}{\Gamma(b) \Gamma(c-b)} \int_{0}^{1} t^{b-1}(1-t)^{c-b-1}(1-z t)^{-a} d t
$$


valid for $\operatorname{Re} c>\operatorname{Re} b>0,|z|<1$, as was done in a similar case in a. $\beta$. Thus we have proved that $a_{m}(\lambda)$ depends on $\lambda$ regularly, if $\lambda \in D_{0}$.

Keeping $\epsilon, 0<\epsilon<\delta$ fixed, we choose a compact set $C \subset D_{0}$. Then we have $\left|F_{2}(\lambda, \epsilon, m)\right|<N_{1}(m=1,2, \cdots)$, with an $N_{1}$ depending on $C$ only. Furthermore

$$
\left|F_{1}(\lambda, \epsilon, m)\right| \leqq \frac{m}{\pi} \int_{0}^{\epsilon} \phi(1-\cos \phi)^{-3 / 4}\left|\Psi_{1}(\phi, \lambda)\right| d \phi<m N_{2},
$$

where $N_{2}$ again depends on $C$ only. Hence we get $\left|a_{m}(\lambda)\right|<K m(m=1,2, \cdots)$ for any fixed compact set $C \subset D_{0}$, with $K$ independent of $\lambda$ and $m$.

For later use observe that for each fixed $m a_{m}(\lambda)$ remains bounded if $\lambda$ tends to $1 / 4$ from the left along the real line. Thus if

$$
f(\phi)=\sum_{-\infty}^{\infty} a_{m} e^{i m \phi}
$$

the series

$$
h_{f}(\lambda)=\sum_{-\infty}^{\infty} a_{m}(\lambda) \bar{a}_{m}\left(\lambda_{\sigma, \tau}(m)\right)
$$

represents an analytic function in $D_{0}$, which for $\operatorname{Im} \lambda>0$ coincides with $(R(\lambda) f, f)_{0, \pi}$.

Hence the proof of the statement made at the beginning of the present section $(\beta)$ has been completed.

$(\gamma)$ As the final step, we consider the spectrum of $Q_{0}^{-}$in the interval $[1 / 4,+\infty)$. The result is as follows: The spectrum of $Q_{0}^{-}$fills out the interval $[1 / 4,+\infty)$ and is equivalent to the Lebesgue measure. Its spectral resolution is continuous at the point $1 / 4$. This is a consequence, in the same way as in a.r of the following fact: For any $f \in C \cap H_{\sigma, \tau}^{-}$there exists a function $h_{f}(\lambda)$ which coincides with $(R(\lambda) f, f)_{\sigma, \tau}$ for $\operatorname{Im} \lambda>0$, and which is regular at each point of the interval $(1 / 4,+\infty)$. To prove this statement, we again consider, as above in $(\beta)$, the functions $a_{m}(\lambda)(m=1,2, \cdots)$ for a fixed $\lambda_{0}, 1 / 4<\lambda_{0}<+\infty$. Then one has to show first that they are regular functions of $\lambda$ in a common neighborhood around $\lambda_{0}$, and secondly that the rate of growth of the sequence $\left|a_{m}(\lambda)\right|$, if $\lambda$ is restricted to this neighborhood, is not faster than a fixed power of $m$. To establish all this the reasonings of $(\beta)$ require only trivial modifications, so that we omit further details. Similarly as in a. $\gamma$ the only thing we have to take care of is that $\operatorname{Re} \mu$ changes its sign when $\lambda$ crosses the real line between $1 / 4$ and $+\infty$ in the direction of the half-plane $\operatorname{Im} \lambda<0$. But all considerations of $(\beta)$ remain valid if $\operatorname{Re} \mu>-\gamma$, with a sufficiently small fixed positive $\gamma$; but this condition is satisfied if $\lambda$ remains sufficiently close to $\boldsymbol{\lambda}_{\mathbf{0}}$.

Finally, making use of the remark made in $(\beta)$, according to which for 
each fixed $m\left|a_{m}(\lambda)\right|$ remains bounded, if $\lambda \rightarrow 1 / 4-0$ along the real line, we may prove as in a. $\gamma$ that $1 / 4$ cannot be an eigenvalue for $Q_{0}^{-}$.

Thus the proof of the statement concerning the structure of $Q_{0}$, made at the beginning of $\mathrm{B} 4$, has been completed.

Hence, combining the results of II.A and II.B with Corollary A to Theorem I, we get the following:

THEOREM II. Let $T_{a}$ be the Kronecker product of two irreducible representations of $L_{3}$ taken from the continuous series $C_{q}^{0}$ and $E_{q}$, with the Casimir operators $q_{1} I$ and $q_{2} I\left(0<q_{1}, q_{2}<+\infty\right)$ resp. Then $T_{a}$ is a direct sum of two representations $T_{a}^{c}$ and $T_{a}^{d}$. If $\operatorname{Re}(\sigma+\tau) \leqq 1 / 2$, where $\sigma=\left(1 / 4-q_{1}\right)^{1 / 2}$ and $\tau=\left(1 / 4-q_{2}\right)^{1 / 2}$, then $T_{a}^{c}$ is a two-fold copy of a representation, which is a continuous direct sum, with respect to a weight function equivalent to the Lebesgue measure, of all representations of the principal continuous series; otherwise the direct sum of a representation of the previous type with an irreducible representation of the type $E_{q_{0}}$, where $q_{0}=1 / 4-(\sigma+\tau-1 / 2)^{2} . T_{a}^{d}$ is a discrete direct sum of irreducible representations of the type $D_{s}^{+}, D_{s}^{-}(s=1,2, \cdots)$, each of them occurring once.

\section{BIBLIOGRAPHY}

1. V. A. Bargmann, Irreducible unitary representations of the Lorentz group, Ann. of Math. vol. 48 (1947) pp. 568-640.

2. E. T. Copson, An introduction to the theory of functions of a complex variable, Oxford, Clarendon Press, 1955.

3. I. M. Gel'fand and M. A. Nalmark, Unitary representations of the Lorentz group, Izv. Acad. Sci. SSSR vol. 11 (1947) pp. 411-504 (Russian).

4. G. W. Mackey, Induced representations of locally compact groups. I, Ann. of Math. vol. 55 (1952) pp. 101-139.

5. - Induced representations of locally compact groups. II, Ann. of Math. vol. 58 (1953) pp. 193-220.

6. F. I. Mautner, Unitary representations of locally compact groups. II, Ann. of Math. vol. 52 (1950) pp. 528-556.

7. - On the decomposition of unitary representations of Lie groups, Proc. Amer. Math. Soc. vol. 2 (1951) pp. 480-485.

8. F. J. Murray and J. von Neumann, On rings of operators. I, Ann. of Math. vol. 37 (1936) pp. 116-229.

9. J. von Neumann, On rings of operators. Reduction Theory, Ann. of Math. vol. 50 (1949) pp. 401-485.

10. M. A. NaImark, Decomposition of a tensorial product of irreducible representations of the proper Lorentz group into irreducible representations, Trudy Moskov. Mat. Obšc. vol. 8 (1959) pp. 121-153.

11. I. E. Segal, A class of operator algebras, which are determined by groups, Duke Math. J. vol. 18 (1951) pp. 221-265.

12. - Hypermaximality of certain operators on Lie groups, Proc. Amer. Math. Soc. vol. 3 (1952) pp. 13-15.

13. E. C. Titchmarsh, Eigenfunction expansions associated with second-order differential equations, Oxford, Clarendon Press, 1946.

RIAS,

BALTIMORE, MARYLAND 